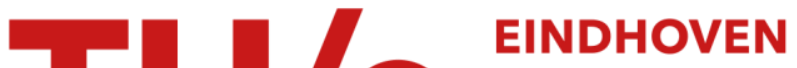 UNIVERSITY OF TECHNOLOGY
}

\section{Modelling recrystallization and grain growth of tungsten induced by neutron displacement defects}

\section{Citation for published version (APA):}

Mannheim, A., van Dommelen, J. A. W., \& Geers, M. G. D. (2018). Modelling recrystallization and grain growth of tungsten induced by neutron displacement defects. Mechanics of Materials, 123, 43-58.

https://doi.org/10.1016/j.mechmat.2018.04.008

DOI:

10.1016/j.mechmat.2018.04.008

Document status and date:

Published: 01/08/2018

\section{Document Version:}

Accepted manuscript including changes made at the peer-review stage

\section{Please check the document version of this publication:}

- A submitted manuscript is the version of the article upon submission and before peer-review. There can be important differences between the submitted version and the official published version of record. People interested in the research are advised to contact the author for the final version of the publication, or visit the $\mathrm{DOI}$ to the publisher's website.

- The final author version and the galley proof are versions of the publication after peer review.

- The final published version features the final layout of the paper including the volume, issue and page numbers.

Link to publication

\section{General rights}

Copyright and moral rights for the publications made accessible in the public portal are retained by the authors and/or other copyright owners and it is a condition of accessing publications that users recognise and abide by the legal requirements associated with these rights.

- Users may download and print one copy of any publication from the public portal for the purpose of private study or research.

- You may not further distribute the material or use it for any profit-making activity or commercial gain

- You may freely distribute the URL identifying the publication in the public portal.

If the publication is distributed under the terms of Article 25fa of the Dutch Copyright Act, indicated by the "Taverne" license above, please follow below link for the End User Agreement:

www.tue.nl/taverne

Take down policy

If you believe that this document breaches copyright please contact us at:

openaccess@tue.nl

providing details and we will investigate your claim. 


\title{
Modelling recrystallization and grain growth of tungsten induced by neutron displacement defects
}

\author{
A. Mannheim, J.A.W. van Dommelen* and M.G.D. Geers \\ Mechanics of Materials, Mechanical Engineering, Eindhoven University of \\ Technology, P.O. Box 513, 5600 MB Eindhoven, The Netherlands
}

April 4, 2018

\begin{abstract}
A multi-scale model is developed for the long-term microstructural evolution of tungsten under cascade damage conditions and for different irradiation temperatures as they may occur in a divertor of a nuclear fusion reactor. In particular the competition between damage accumulation and recovery processes, including grain growth and recrystallization are captured. A mean-field model for recrystallization and grain growth is coupled to a cluster dynamics model for the evolution of the neutron damage. The displacement damage is produced in the form of defect clusters, for which a parameterized temperature-dependent scaling law is implemented. A physically-based grain nucleation model is implemented in the mean-field recrystallization model. The competition between the various temperature dependent mechanisms and their effects on the evolving microstructure is studied in the range of $T=1000{ }^{\circ} \mathrm{C}-1400{ }^{\circ} \mathrm{C}$.
\end{abstract}

\section{INTRODUCTION}

The use of nuclear fusion for generation of electricity could help the world to meet the energy demands and bridge the next few hundred years on the way to $100 \%$ renewable energy generation. For the economical viability of fusion reactors, divertors (components used as heat extractor, armour and He-absorber [1]) need to have a sufficient lifetime (at least 2 years of operation before replacement) and are therefore critical parts of the reactor. Understanding the degradation and recovery mechanisms for this component (likely to be made of tungsten [2]) is essential. It is thereby important to look beyond the surface effects $(\sim 10 \mu \mathrm{m})$ of the heat, neutron- and ion-irradiation on the material and to take into account the evolution of the microstructure and thermomechanical properties in the entire tungsten monoblock.

Due to the large heat loads $\left(>10 \mathrm{MW} / \mathrm{m}^{2}[3]\right)$ and active cooling, the divertor has to endure a high surface temperature and temperature gradient. This leads to large stresses and possibly cracking [4-6]. The thermomechanical properties (such as thermal conductivity and mechanical behavior) evolve during fusion operation, as they depend on the evolving microstructure. The divertor can still be considered to fulfill its functions as long as (1) the heat is still adequately conducted towards the cooling water, (2) it acts as an armor, keeping the plasma ions away from the cooling water and other underlying components, and (3) it is structurally sufficiently intact [7].

In the bulk of the divertor monoblock, the main loads that will affect the microstructure are a neutron load $\left(\sim 10^{-6} \mathrm{dpa} / \mathrm{s}\right.$ for ITER) [8] in combination with high temperatures (approximately in the range of $400^{\circ} \mathrm{C}-1000^{\circ} \mathrm{C}$ or higher. The minimum temperature is set by the

\footnotetext{
*Address correspondence to J.A.W. van Dommelen, Mechanics of Materials, Mechanical Engineering, Eindhoven University of Technology, P.O. Box 513, 5600 MB Eindhoven, The Netherlands. Electronic mail: j.a.w.v.dommelen@tue.nl
} 
cooling water and the maximum is set by the heat input, the thermal conduction coefficient, the cooling and the geometry of the monoblock. The maximum allowable temperature is the temperature at which the mechanical integrity can be presented. The fast neutrons will (apart from transmutations) generate large displacement cascades, causing lattice damage. On a microstructural level, this means that a high stored energy is built up in the lattice, in addition to an initial dislocation density. Plasma ions (mainly $\mathrm{H}$ and $\mathrm{He}$ ) will damage the surface of the divertor. $\mathrm{H}$ and $\mathrm{He}$, as well as Re and Os are also introduced into the material by transmutations.

The combination of a high temperature and a high level of displacement damage will induce recovery and grain growth [9-13]. Moreover, recrystallization may take place, since tungsten with a high dislocation density is known to recrystallize under high temperature annealing [1416]. Grain growth can be both thermally-induced [17] or irradiation-induced [9-13]. Recrystallization of polycrystalline materials has also been observed to be enhanced by irradiation [18-20], and it is conceivable that neutron cascade damage lowers the recrystallization temperature [9] of tungsten (normally in the order of $1300^{\circ} \mathrm{C}[7]$ ). Moreover, under fusion operation, the thermal conductivity of the tungsten monoblock may decrease as a consequence of the point defects, dislocation loops, transmutations and gas bubbles that will be formed [21]. This implies that the temperature gradient over the monoblock may increase, and the maximum temperature in the block may become higher as well, increasing the likelihood of grain growth and recrystallization to occur (and increasing the thermal stresses, erosion due to sputtering, creep and high temperature embrittlement [22]). The effects mentioned above may be aggravated by the high power transient heat loads that are expected to occur as a consequence of instabilities in the plasma [23].

The consequences of recrystallization and grain growth for the material properties of tungsten can be positive or negative. Both these recovery mechanisms lead to a decrease in the displacement defect density in the material and therefore also a decrease in strength. Recrystallization and grain growth may also lead to a speed-up of the segregation of pre-existing and radiation-induced impurities (including neutron-induced transmutations). In some cases, recrystallization embrittlement is reported for tungsten [24], although an improved ductility after recrystallization has also been observed [25]. The recrystallized grain size may be of influence for the ductility of tungsten $[26,27]$, especially since the grain boundaries of tungsten seem to be the weakest point and intergranular fracture is the most common failure mechanism for tungsten [28].

Modelling approaches for static and dynamic recrystallization (where recrystallization occurs under annealing, respectively after and during applying a load on the material $[17,29]$ ) range from simple empirical laws [30] up to computationally complex models that can take into account the topology [31, 32], such as the Monte Carlo-Potts, Cellular Automata, Phase-Field, Vertex and Level Set methods. The topological models can be used to simulate local physical phenomena (such as the evolution of the grain boundary shape [33] and the interaction with second-phase particles [34]), as well as local variations in the grain boundary mobility and grain boundary energy. In between are the (computationally efficient) mean-field models [35-38], some of which consider only the evolution of the average values of the grain size and the defect density whereas in $[36,38]$ their distributions are also considered.

Nucleation of new grains usually occurs near grain boundaries, triple junctions and twins [17] and may occur at sites with a large gradient in the dislocation density [39]. It is difficult to observe the precise nucleation mechanisms experimentally. Based on the activation energy for nucleation, the nuclei emerge from an existing structure, such as a subgrain. Bulging is the nucleation mechanism for formation of new grains that is most reported in literature. This can be accompanied by twinning $[29,39,40]$. For neutron-induced recrystallization, it was suggested that instead of subgrains, interstitial dislocation loops may be the preceding structure leading to nucleation [41]. A deformed crystal structure may have to rearrange (first creating more ordered dislocations structures) before nucleation sites are formed [29].

A basic analytical model for recrystallization that employs a constant nucleation rate is the Kolmogorov-Johnson-Mehl-Avrami model [17]. Most empirical models do not incorporate explicit nucleation events, as usually the final average grain size is predicted and not the size 
evolution in time. [31]. Most models that do treat nucleation events explicitly, employ a critical dislocation density or critical (plastic) strain [31,38,42-44] that depends on the material parameters and process parameters, including the effective plastic strain rate. When nucleation is modelled in more detail, nuclei are often formed out of subgrains [36, 45].

Recrystallization and grain growth are driven by the stored lattice energy in the material. For neutron-irradiated polycrystalline tungsten, the lattice energy is stored in the form of pre-existing lattice defects, displacement defects and grain boundaries. Neutron-irradiated or ion-irradiated tungsten is experimentally observed to contain a wide variety of types of displacement damage, such as vacancies, self-interstitial atoms (SIAs), dislocations, prismatic dislocation loops of vacancy/SIA type, vacancy clusters, voids and SIA-clusters [13, 46-49].

The energetic fusion neutrons create a high amount of displacement defects in a single cascade, leading to a local defect distribution [50]. The damage resulting from displacement cascades is known to be strongly temperature-dependent [51]. The displacement defects interact with the dislocation network, which influences the dislocation density $[52,53]$. The evolution of the defect size distribution in tungsten under neutron and/or helium irradiation has been modelled using Cluster Dynamics (CD) [8,54-62] and Object-Oriented Kinetic Monte Carlo methods (OKMC) [63-66], where most studies have concentrated on the influence of $\mathrm{He}$ on the clustering behaviour. Kinetic and energetic parameters for these models have been determined using Molecular Dynamics (MD) [51, 59, 64, 67, 68] and Density-Functional Theory (DFT) [69].

Cluster dynamics is a suitable technique for modelling the evolution of irradiation damage for higher irradiation dosages and longer time scales. It provides reasonably good results, as becomes clear from the comparion between $\mathrm{CD}$ and $\mathrm{kMC}$ simulations [70,71], or the comparison between simulation and experimental results [72]. However it is important to keep in mind that cluster dynamics has its limits. For one, experimental observations show that $1 \mathrm{D}$ void lattices form under irradiation (for tungsten, it has been shown under fission-neutron irradiation [73], and for many other materials it has been shown with fission neutrons and protons and electrons, see [74], page 386). Contrary to the cluster dynamics model, the production bias model [74] takes into account the 1D-migration of various SIA-clusters in the direction of their Burgers vector. Moreover, swelling does not occur homogeneously: the swelling rates are observed to be a function of the void density and dislocation density and more swelling happens at the interior of the grain, and not in the same way as is predicted by the cluster dynamics model [74].

In this paper, a framework for modelling recrystallization and grain growth under irradiation condition is presented. A mean-field approach is used for modelling recrystallization, for which a physics-based nucleation model is implemented. Grain growth and recrystallization are driven by the neutron-induced displacement defects, which are modelled using cluster dynamics. The ability of the model to capture the microstructural evolution (in the form of grain size evolution and the evolution of the defect densities) is assessed for various temperatures, using small cluster sizes. The influence of the initial grain size and temperature on the competing mechanisms is analyzed. The study concludes by discussing the most dominant mechanisms at different temperatures (defect accumulation, grain growth, nucleation). 


\section{Multi-scale microstructure evolution model}

\subsection{Two-scale approach}

A multi-scale model is used to describe the microstructural evolution of tungsten under heat and neutron irradiation at various temperatures. Recrystallization, recovery and grain growth, driven by the stored lattice energy, are modelled using a mean-field method, based on [38]. The recrystallization model describes the material's structure at a scale of $\mu \mathrm{m}-\mathrm{mm}$. The stored lattice energy follows from the continuous generation, evolution and annihilation of lattice defects inside each of the grains (lengthscale $10^{-1}-10^{2} \mathrm{\mu m}$ ), which is modelled in a coupled manner for a set of grains that represent the microstructure, using a cluster dynamics approach [8] in the mean-field recrystallization model. This coupling is illustrated in Figure 1. In both models, the current grain size and defect density determine the subsequent evolution of the microstructure. As a consequence of grain growth and recrystallization, defects are swept and removed, reducing the defect density inside the grain. These processes also affect the grain size. In the cluster dynamics model, the grain size determines the diffusion distance of the defects from the core of the grain to the grain boundaries, which act as sinks.

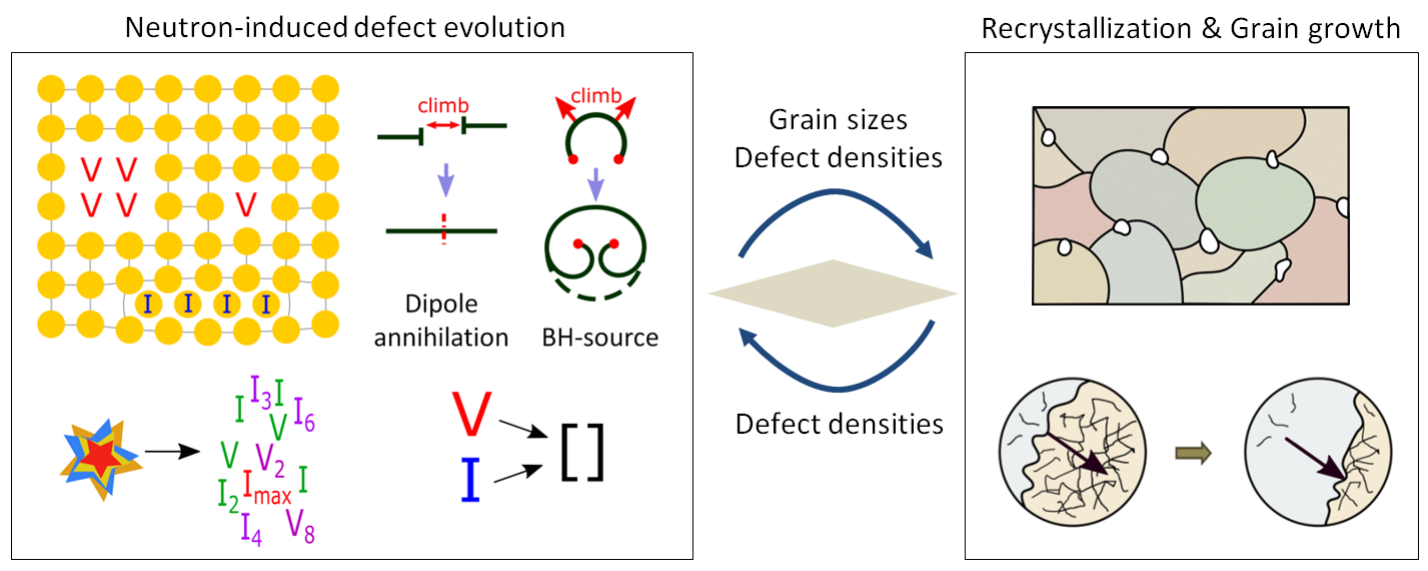

Figure 1: Sketch of the two-scale model for microstructural evolution under lattice defect generation, grain growth and recrystallization. Left: lattice vacancy and self-interstitial defects and their clusters are generated (bottom, left), annihilated (bottom, right) and evolved (top, left). Changes in the network dislocation density due to dislocation climb in the form of dipole annihiliation and Bardeen-Herring sources $[52,75]$ are taken into account (top, right). Right: recrystallization and grain growth are modelled with the processes of nucleation and grain boundary migration. Centre: the two models are coupled through defect densities and grain sizes.

\subsection{Recrystallization model}

A mean-field model is proposed to describe recrystallization as well as grain size evolution by grain growth, based on the work of Bernard et al. [38]. The microstructure is represented by sets of representative grains, which interact with the surrounding (averaged) media. The mean-field model uses two homogeneous equivalent media (HEMs), so that the effects of grains with a high defect energy (HD) and low defect energy (LD) can be distinguished. In this model, the grains are assumed to be spherical. Each representative grain $k$ has the following properties: a radius $r_{k}$, the number of grains $N_{k}$ it represents, a dislocation defect density $\rho_{k}$ and cluster defect concentrations $C_{k}$ for each type of defect. The properties of the media are computed by taking the volume average of the properties of the corresponding grain sets [38]. The representative grains are categorized into the LD- or HD-medium based on their volumetric stored defect energy. 


\subsubsection{Grain growth}

The grain size evolution of a grain $k$ during a time increment $\Delta t=t_{n+1}-t_{n}$ is determined from the volume changes $\Delta V_{k}$ of the grain as a result of the velocity of the grain boundary $v_{k}$ :

$$
\Delta V_{k}=4 \pi r_{k}^{2} \Delta r_{k}=4 \pi r_{k}^{2} v_{k} \Delta t
$$

The grain boundary velocity depends on the grain boundary mobility $m$ and on the driving force ${ }^{1} \Delta E[38]:$

$$
v_{k}=m \Delta E_{k}^{H E M}=m\left[E^{H E M}-E_{k}\right]=m\left[E^{V, H E M}-E_{k}^{V}+\frac{3}{2} \gamma_{b}\left(\frac{1}{r^{H E M}}-\frac{1}{r_{k}}\right)\right] .
$$

Here, the superscript ${ }^{V}$ denotes volume, $E_{k}^{V}$ is the volumetric part of the stored energy of representative grain $k, \gamma_{b}$ is the grain boundary surface energy, $r_{k}$ is the radius of representative grain $k$ and $r^{H E M}$ is the equivalent radius of the HEM (where HEM stands for the LD- or HD-medium) and $E^{V, H E M}$ is the volume averaged stored energy of the representative grains in the HEM; i.e. the grain radius corresponding to the average GB surface area, for which the total GB-surface area is divided by the total number of grains.

\section{Surface fractions}

For each time increment, the volume change of each representative grain is computed. The grains grow or shrink with respect to each HEM, as illustrated in Figure 2. The grain boundaries between an LD-grain and the LD-HEM will be less mobile than the mixed boundaries (LD-grain and HD-HEM or HD-grain and LD-HEM). The surface fraction $\phi^{L D}$ denotes the mobile fraction of the total surface area of an LD-grain which is the part of the grain boundary that is shared with the HD-HEM and similarly $\phi^{H D}$ denotes the mobile surface fraction of an HD-grain (i.e. the part shared with the LD-HEM).
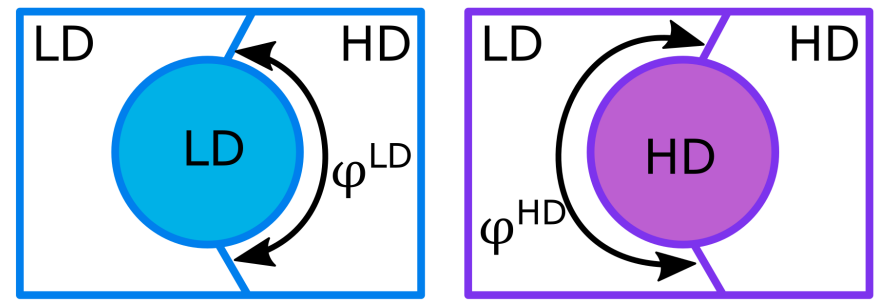

Figure 2: The mobile fractions of the grain boundary surface area $\phi^{L D}$ and $\phi^{H D}$ for LD- and HD-grains. Adapted from [38].

\section{Volume changes}

Following [38], the volume changes $\Delta V_{i}$ for each HD-grain $i$ and $\Delta V_{j}$ for LD-grain $j$ in a time increment $\Delta t$ are given by

$$
\begin{aligned}
& \Delta V_{i}=\phi^{H D} \Delta V_{i}^{H D / L D}+\left(1-\phi^{H D}\right) \Delta V_{i}^{H D / H D} \\
& \Delta V_{j}=\phi^{L D} \Delta V_{j}^{L D / H D}+\left(1-\phi^{L D}\right) \Delta V_{j}^{L D / L D},
\end{aligned}
$$

with $\phi^{H D} \Delta V_{i}^{H D / L D}$ the volume change between the HD-grain $i$ and LD-HEM and so forth. Volume conservation dictates [38] that:

1. The volume transfer to/from the media should be equal: $\sum_{i} \phi^{H D} \Delta V_{i}^{H D / L D}=-\sum_{j} \phi^{L D} \Delta V_{j}^{L D / H D}$ From this it follows for the surface fractions that [38]: $\phi^{H D}=-\phi^{L D} \frac{\sum_{j \in L D} N_{j} r_{j}^{2} \Delta E_{j}^{L D / H D}}{\sum_{i \in H D} N_{i} r_{i}^{2} \Delta E_{i}^{H D / L D}}$.

\footnotetext{
${ }^{1}$ If the grain radius is $r$, then the density of grain boundary surface per unit volume is $\frac{1}{2} \frac{4 \pi r^{2}}{4 \pi r^{3} / 3}=\frac{3}{2 r}$, where $\frac{1}{2}$ avoids counting every GB-surface area twice.
} 
2. All interactions of a HEM with each grain within this HEM should not lead to a net volume change. For the LD-HEM, this means that:

$\sum_{\left\{j \mid \Delta E_{j}^{L D / L D}>0\right\}} N_{j} \Delta V_{j}^{L D / L D}=\sum_{\left\{j \mid \Delta E_{j}^{L D / L D}<0\right\}} N_{j} \Delta V_{j}^{L D / L D}$.

This is accounted for in the following way [38]: first the new volumes of the LD-grains that grow by interactions within the set are computed. Next, the total volume corresponding to LD-LD shrinkage is computed in such a way that it equals the total LD-LD growth volume.

In this model, necklace-type nucleation is assumed, therefore the LD surface fraction is given by [38]:

$$
\phi^{L D}=1-\left(f^{L D}\right)^{2 / 3}
$$

where $f^{L D}$ is the fraction of the total volume that belongs to the LD-set. Experimental findings indicate that in the case of static recrystallization of tungsten, most new grains emerge at the grain boundaries [76]. Not much is known about the nucleation mechanism induced by particle irradiation, but for $\mathrm{U}_{10} \mathrm{Mo}$, it was observed that nucleation occured predominantly at grain boundaries [20]. In case of homogeneous nucleation, the surface fractions are given by $\phi^{L D}=f^{L D}$ and $\phi^{H D}=1-f^{L D}[38]$.

\section{Defect density evolution for growing grains}

Moving grain boundaries sweep lattice defects and dislocations [38]. The volume that is added to the growing grains therefore only contains equilibrium defect concentrations. Note that the grain boundary movements with respect to the HD- and LD-media can lead to both growth and shrinkage of the same grain within one time increment. Let $V^{*}$ denote the volume of the grain after the GB-movements due to shrinkage only (LD/LD-HEM shrinkage and LD/HD-HEM shrinkage). The updated defect concentration $C_{i}\left(t_{n+1}\right)$ (or: density $\rho_{i}\left(t_{n+1}\right)$ ) of defect type $i$ of a growing grain can be computed from:

$$
V\left(t_{n+1}\right) C_{i}\left(t_{n+1}\right)=V_{s}\left(t_{n+1}\right) C_{i}\left(t_{n}\right)+\left(V\left(t_{n+1}\right)-V^{*}\left(t_{n+1}\right)\right) C_{i}^{0},
$$

where $C_{i}^{0}$ is the equilibrium defect concentration, and $V\left(t_{n+1}\right)$ the grain volume at $t_{n+1}$. The defect concentrations in shrinking grains are assumed not to be influenced by the grain boundary movement.

\section{Volumetric stored energy}

For the volumetric part of the stored energy $E_{k}^{V}$ of a grain $k$, the stored defect energy and the configurational entropy $S$ are taken into account:

$$
E_{k}^{V}=\sum_{i=1}^{N_{d}}\left[C_{i} E_{i}^{f}\right]-T S
$$

Here, $N_{d}$ is the amount of different types of defects in the material, $C_{i}$ is the concentration of defect type $i, E_{i}^{f}$ is the formation energy of defect type $i$ and $T$ is the temperature. The calculation of the configurational entropy is given in Appendix A.

\section{Grain boundary mobility}

The grain boundary mobility $m$ is described, using the temperature dependency from the Turnbull estimate $[36,77,78]$ :

$$
m(T)=K_{m} \frac{\beta \delta V_{a t}}{b^{2} R T} D_{0}^{G B} \exp \left(\frac{-Q^{G B}}{k_{B} T}\right)
$$

Here, $K_{m}$ is a material parameter, $\delta$ is the thickness of the grain boundary, $\beta$ the action parameter (here taken 0.3), $V_{a t}$ the atomic volume, $R$ the gas constant, $T$ the temperature $(\mathrm{K}), k_{B}$ the Boltzmann constant, $D_{0}^{G B}$ the self-diffusivity of tungsten along grain boundaries and $Q^{G B}$ the activation energy for diffusion of tungsten along grain boundaries (see Table 7). The grain boundary mobility is taken identical for all grains, except when grain boundaries are assumed to be pinned. This is further detailed in section 3.1. 


\subsubsection{Nucleation}

\section{Nucleation rate}

During recrystallization, new defect-free grains can form at the cost of old defected grains when this is energetically favourable and kinetically achievable. The nucleation rate $\dot{N}_{c}$ is computed using

$$
\dot{N}_{c}=K_{N} A_{n u c} \exp \left(\frac{-E_{a c t}}{k_{B} T}\right) \exp \left(\frac{-Q^{G B}}{k_{B} T}\right) .
$$

Here, $A_{n u c}$ is the total surface area that is available for (necklace) nucleation, $K_{N}$ is a rate constant and $E_{a c t}$ is the activation energy for forming a stable grain.

\section{Nucleus radius and activation energy}

In classical nucleation theory (CNT), a stable nucleus has an initial radius such that the Gibbs free energy change $\Delta G$ decreases with further growth: $\frac{\partial \Delta G}{\partial r}<0$. CNT is used as a starting point, where the following expression is used for the Gibbs free energy change:

$$
\Delta G=\frac{1}{K_{a}}\left[\frac{4 \pi r^{3}}{3}\left(E_{0}^{V}-E^{V}\right)+3 \pi r^{2} \gamma_{b}\right],
$$

where a fitting parameter $K_{a}$ is introduced, because it is known that the activation energy for the formation of a recrystallized grain is smaller than what CNT predicts [17]. Because of necklacetype nucleation, a grain boundary energy of $3 \pi r^{2} \gamma_{b}$ is used $\left(4 \pi r^{2}\right.$ of new surface area is formed, and this is diminished by an intersection of $\pi r^{2}$ that is lost). $E_{0}^{V}$ is the volumetric stored energy of the nucleated grain and $E^{V}$ is the current volumetric stored energy in the volume that will trigger nucleation. It is assumed that nucleation always initiates at the boundary of an HDgrain, but that the volume for the nucleus consists of both HD- and LD-grains, with the volume fractions following the surface fractions. This implies that nuclei can be formed at HD/HD-grain boundaries and $\mathrm{HD} / \mathrm{LD}$-grain boundaries, but not at $\mathrm{LD} / \mathrm{LD}$-grain boundaries. It also implies that $E^{V}$ is given by:

$$
E^{V}=\phi^{L D} E^{V, H D}+\left(1-\phi^{L D}\right) E^{V, L D},
$$

where $E^{V, H D}$ and $E^{V, L D}$ are the volumetric parts of the averaged stored energy in the HD- and LD-media. Based on the condition $\partial \Delta G / \partial r=0$, a static solution for the critical radius $r^{*}$ can be obtained. However, since the value of $\Delta G$ will change during the process of recrystallization and irradiation $\left(E^{V}\right.$ changes over time), the stability condition for a grain, apart from the growth condition for this nucleus, is $\frac{d \Delta G}{d t}<0$ :

$$
\frac{d \Delta G}{d t}=\frac{\partial \Delta G}{\partial r} \frac{d r}{d t}+\frac{\partial \Delta G}{\partial E^{V}} \frac{d E^{V}}{d t}<0 .
$$

Here, $\frac{d r}{d t}$ is the growth rate of the nucleus after it has formed, given by: $\frac{d r}{d t}=m\left(E-E_{0}\right)$, with $E_{0}$ the energy of the nucleated grain and $E$ the average total energy of both media together, weighted with the surface fractions: $E=\phi^{L D} E^{H D}+\left(1-\phi^{L D}\right) E^{L D}$ (where $E=E^{V}+E^{S}$, the total energy is the sum of the volumetric and surface parts of the energy). After some rewriting:

$$
\frac{\partial \Delta G}{\partial t}=\frac{1}{K_{a}}\left[-\frac{4 \pi}{3} r^{3} \frac{d E^{V}}{d t}-4 \pi r^{2} m\left(E^{V}-E_{0}^{V}\right)\left(E-E_{0}^{V}\right)+6 \pi r m \gamma_{b}\left(E^{V}+E-2 E_{0}^{V}\right)-9 \pi m \gamma_{b}^{2}\right]<0
$$

The value of $\frac{d E^{V}}{d t}$ is determined numerically. The radius of the nucleus $r_{n u c}$ is then obtained from the condition $\partial \Delta G / \partial t=0$. The activation energy for nucleation $E_{a c t}$ is then given by $\Delta G\left(r_{n u c}\right)$ if $r_{n u c}<r^{*}$, and otherwise by $\Delta G\left(r^{*}\right)$. The volume for nucleation is delivered by the existing grains, weighted with their respective surface fractions. The volume $\operatorname{loss} \Delta V_{i}^{H D}$ for a representative HD-grain $i$ due to a nucleation volume $V_{n u c}$ is

$$
\Delta V_{i}^{H D}=\phi^{L D} V_{n u c} \frac{4 \pi r_{i}^{2}}{\sum_{i \in H D} 4 \pi N_{i} r_{i}^{2}}
$$

Likewise, $\Delta V_{j}^{L D}$ for an LD-grain $j$ is such that $\sum_{j \in L D} N_{j} \Delta V_{j}^{L D}=\left(1-\phi^{L D}\right) V_{n u c}$. 


\subsection{Defect evolution model}

The evolution of the lattice damage under irradiation inside each representative grain is modelled using a cluster dynamics-based approach. For the evolution of the displacement defects (vacancies, self-interstitial atoms and their clusters), the model of Li et al. [8] is used as a basis. The evolution of these displacement defects also leads to changes in the dislocation density, which are modelled here using the work of Stoller [52]. To accommodate for neutron cascade damage, a damage generation term for the displacement defects is used, exploiting the work of Yi et al. [46], where the temperature-dependence of the primary damage [51] (the displacement damage that remains at several picoseconds after the cascade started) is also taken into account.

\subsubsection{Evolution processes}

The following set of rules describes the types of displacement defects that are considered and the processes that induce changes in their concentrations [8]:

1. Defect types: vacancies $\left(V_{1}\right)$, self-interstitial atoms $\left(I_{1}\right)$, their clusters $V_{n}$ and $I_{n}$ (where $n=2 \ldots N_{\max }$ denotes the size of the cluster in terms of the number of point defects involved) and dislocation network lines;

2. All displacement defects are considered to be immobile, except for $V_{1}, I_{1}$ and $I_{2}$ [8];

3. All types of clusters of displacement defects can be generated as primary damage and they can change in size by absorption/emission of mobile point defects (or mobile clusters);

4. Interstitial defect clusters can absorb and emit all types of mobile defects; however the emission of $I_{1}$ and $I_{2}$ from vacancy clusters is neglected;

5. The mobile displacement defects can be annihilated by absorption at defect sinks (dislocation lines and grain boundaries) and by recombination.

Furthermore, the dislocation density $\rho$ can increase by pinned dislocation segments/lines that may act as Bardeen-Herring sources (which are able to generate more dislocations via climb) [52] or $\rho$ can decrease by dipole annihilation.

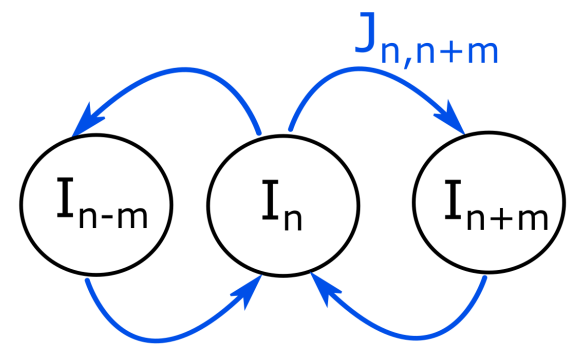

Figure 3: The cluster $I_{n}$ can change in size by absorption/emission of mobile defects of size $m$. The defects $V_{1}, I_{1}$ and $I_{2}$ are considered mobile. For example flux $J_{n, n+m}$ can be associated with the amount of clusters that change from size $n$ into size $n+m$ per second.

\subsubsection{Chemical rate equations}

All the processes that are described above (generation, absorption, emission, ...) are captured in a set of $2 N_{\max }+1$ equations that describe the coupled evolution of the defect cluster concentrations [8] and dislocation density [52]:

$$
\begin{aligned}
& \frac{d}{d t}\left[C_{I_{n}}\right]=G_{I_{n}}+\sum_{p}\left[J_{p, n}^{I}-J_{n, p}^{I}\right]-L_{I_{n}} C_{I_{n}} \\
& \frac{d}{d t}\left[C_{V_{n}}\right]=G_{V_{n}}+\sum_{p}\left[J_{p, n}^{V}-J_{n, p}^{V}\right]-L_{V_{n}} C_{V_{n}}
\end{aligned}
$$




$$
\frac{d}{d t}[\rho] \quad=2 \pi v_{c l} S_{B H}-\rho \tau_{c l}^{-1} .
$$

The first equation expresses how the atomic concentration $C_{I_{n}}$ of SIA-clusters $I_{n}$ of size $n$ evolves over time, by defect production $\left(G_{I_{n}}\right)$, various transports $\left(J_{p, n}^{I} / J_{n, p}^{I}\right)$ of mobile defects respectively to/from defect clusters of size $n$ and by removal of mobile defects at sinks of strength $L_{I_{n}}$. The concentration evolution of vacancy clusters $V_{n}$ is calculated analogously. The third equation expresses the evolution of the dislocation density $\rho$. Here $v_{c l}$ is the dislocation climb velocity, $S_{B H}$ is the density of Bardeen-Herring sources [75] and $\tau_{c l}$ is the average lifetime of a dislocation line until dipole annihilation. The complete set of equations can be found in Appendix B and the parameters used in this model can be found in Table 7.

\section{Rate coefficients}

The various absorption and emission rate coefficients are denoted by $k^{+}$and $k^{-}$respectively, so that the absorption fluxes (of the mobile defects to the defect clusters) can be written as $J_{n, p}=k^{+} C_{p-n} C_{n}$; the emission fluxes as $J_{n, p}=k^{-} C_{n}$ and the fluxes to the sinks as: $L C_{n, m o b}=k_{s n k}^{+} C_{n, m o b}$.

The absorption and emission rate coefficients depend on the capture radius $r_{c a p}$ of the cluster, the diffusion coefficient $D_{m o b}$ of the mobile defect and a bias factor $Z$ that accounts for the preferential absorption of a certain type of mobile defect by the specific type of cluster/sink; and in the case of emission, on the binding energy $E^{b}: k_{n}^{+}=2 \pi r_{\text {cap }}(n) Z_{n} D_{m o b}$ (for a 2D-cluster, the prismatic dislocation loops) or $k_{n}^{+}=4 \pi r_{\text {cap }}(n) Z_{n} D_{\text {mob }}$ (for a $3 \mathrm{D}$-cluster, the vacancy clusters) and $k_{n}^{-}=k_{n-1}^{+} \exp \left(-E^{b} / k_{B} T\right) / V_{a t}$.

The absorption rate of mobile defects to the dislocations depends on the dislocation density $\rho$, the dislocation bias factor $Z_{D}^{m o b}$ for preferential absorption and on the diffusion coefficient $D_{m o b}: k_{D+m o b}^{+}=\rho Z_{\text {bias }}^{m o b} D_{\text {mob }}$. The grain boundary sink strength depends on the strengths $S_{m o b}^{s k}$ of all other sinks for the particular type of mobile defect, on the diffusion coefficient $D_{m o b}$ and on the grain size, as $k_{S+m o b}^{+}=S_{m o b}^{s k} 3 D_{m o b} / r_{\text {grain }} . E^{b}$ is the binding energy of the mobile defect to the cluster, which is computed using the capillarity approximation [64]. All the expressions are detailed in Table 4 in Appendix B.

It is assumed that all SIA-clusters $I_{n}$ take the shape of prismatic loops. The capture radius of such a loop is: $r_{c a p}^{I_{n}}=\sqrt{n V_{a t} / \pi b}$ with $b$ the magnitude of the Burgers vector [8]. All vacancy clusters $V_{n}$ are assumed to be spherical, which gives a capture radius of $r_{c a p}^{V_{n}}=$ $\left(3 n V_{a t} / 4 \pi\right)^{1 / 3}+\sqrt{3} a_{0} / 4$ where $a_{0}$ is the lattice parameter [8].

\subsubsection{Dislocation density evolution}

When the vacancies or self-interstitial atoms are absorbed/emitted by a dislocation loop or a dislocation segment that is pinned, this dislocation line can bulge out by climb, and grow in length. This is how prismatic dislocation loops are assumed to grow, and how Bardeen-Herring $(\mathrm{BH})$ sources generate more dislocation length by climb. The density of BH-sources is taken to be [52] $S_{B H}=\left(\rho_{p} / 3\right)^{1.5}$, where $\rho_{p}$ is the pinned dislocation density, which is here taken to be $\rho_{p}=0.1 \rho$. The rate at which dislocations are created by BH-sources depends on the climb velocity of the dislocation lines, which is obtained as [52]:

$$
v_{c l}=\frac{2 \pi}{b \ln \left(R / r_{c}\right)}\left(Z_{I} D_{I} C_{I}+2 Z_{I_{2}} D_{I_{2}} C_{I_{2}}-Z_{V} D_{V}\left(C_{V}-C_{V}^{D}\right)\right) .
$$

Here, $C_{V}^{D}$ is the equilibrium vacancy concentration near dislocations, $Z_{I}, Z_{I_{2}}$ and $Z_{V}$ are the dislocation bias factors for each of the mobile defect types, $R$ and $r_{c}$ are the dislocation's outer and core radii. Here, $R / r_{c}$ is taken to be $2 \pi[52] . C_{V}^{D}$ is given by $C_{V}^{D}=C_{V}^{0} \exp \left(\sigma V_{a t} / k_{B} T\right)$ with $\sigma=A \mu b \sqrt{\rho_{p}}[52]$ the internal stress due to the immobilized dislocations, where $C_{V}^{0}$ is the thermal equilibrium vacancy concentration in the bulk, $V_{a t}$ is the atomic volume and $\mu$ is the shear modulus. 
The dislocations, which move due to climb, can annihilate when they meet another dislocation of opposite sign. The lifetime of a dislocation prior to annihilation is, on average, given by $\tau_{c l}=d_{c l} / v_{c l}$ where $d_{c l}=(\pi \rho)^{-1 / 2}[52]$ is the distance that a dislocation can travel prior to annihilation. 


\subsubsection{Displacement defect production rate}

The displacement cascade in the lattice leads to the formation of damage clusters within picoseconds, as a result of the spatial distribution of the defects in combination with a heat spike. In the CD-model, which accounts for the defect evolution at much larger time scales, the formation of clusters of point defects is part of the source term. Here, a power law is used for the relation between cluster size and cluster frequency, following the work of Yi et al. [46], where the temperature dependence is based on MD-simulations and self-ion experiments, as described in [46, 51, 67]:

$$
G_{n}=\frac{A_{\epsilon}}{n^{S_{\epsilon}}} .
$$

Here $G_{n}$ is the frequency of occurence of a defect cluster of size $n$, and $A_{\epsilon}$ and $S_{\epsilon}$ are constants with $\epsilon=I$ or $V$, for the production of interstitial clusters and vacancy clusters respectively. The resulting exponents, obtained by [67] and [46], are listed in Table 1.

\begin{tabular}{|l|l|l|l|}
\hline Sim./Exp. & Energy $(\mathrm{keV})$ & $T(\mathrm{~K})$ & Exponent $S$ \\
\hline$W^{+}$-ions [46] & 150 & 30 & $1.8 \pm 0.1$ \\
\hline$W^{+}$-ions [46] & 400 & 30 & $1.6 \pm 0.2$ \\
\hline MD-sim. [67] & 150 & 0 & $1.63 \pm 0.07$ \\
\hline
\end{tabular}

Table 1: Power law exponents based on two self-ion displacement cascade experiments and a displacement cascade MD-simulation.

No distinction was made between vacancy defects and interstitial defects and these exponents are based on low-temperature cascade results. However, cluster defect production is known to be temperature-dependent [51]. The temperature-dependent defect production rate $G_{0}$ is estimated by weighing the Primary Knock-on Atom (PKA) energy spectrum as expected for DEMO with the number of surviving Frenkel pairs per PKA energy, see Setyawan et al. [51]. The PKAenergy spectrum of Gilbert et al. [79], calculated with the SPECTRA-PKA code for the first wall of DEMO under helium-cooled pebble-bed conditions, is used. For the calculation, the PKA-spectrum is divided into bins, where each bin center corresponds to the PKA-energies of the MD-simulations of Setyawan et al. [51], ranging from $0.1 \mathrm{keV}$ to $100 \mathrm{keV}$. The defect production rates $G_{0}$ thus found at $300 \mathrm{~K}, 1025 \mathrm{~K}$ and $2050 \mathrm{~K}$ are listed in Table 2. The temperature dependency of the defect production rate is observed to be moderate. The values are on the same order of magnitude as estimated by Marian et al. [58] for ITER $\left(G_{0}=5.8 \times 10^{-8} \mathrm{dpa} / \mathrm{s}\right)$.

The temperature dependent exponents $S_{I}$ and $S_{V}$ of the power laws for interstitial cluster production and vacancy cluster production are estimated based on the average unclustered SIAfraction/V-fraction $f_{I}^{U}$ and $f_{V}^{U}$ for each temperature (weighing the number of PKA-events per energy with the unclustered fractions at that energy, using the energy bins described above) and are listed in Table 2. Since the values of the unclustered fractions differ significantly, separate power laws are used for the production rate of vacancy clusters and interstitial clusters. The prefactors $A_{I}$ and $A_{V}$ in the power laws are taken such that for each defect type the total damage production rate equals $G_{0}$, i.e.:

$$
A_{\epsilon}=G_{0} / \sum_{n=1}^{N_{\max }} n^{1-S_{\epsilon}} \quad \epsilon=\{I, V\} .
$$

In the model, linear interpolation is used to determine $G_{0}, S_{I}$ and $S_{V}$ at temperatures different from $300 \mathrm{~K}, 1025 \mathrm{~K}$ or $2050 \mathrm{~K}$.

The production rate $G_{0}$ is taken as the production rate in a material with only a low density of displacement defects. When, over time, cascades occur in areas in the material that already contain a lot of defects, the net defect production rate decreases. As a first order approximation, it is assumed that the defect production rate $G$ depends linearly on the fraction $f_{D}$ of the atomic sites in the material containing defects, i.e. $G=G_{0}\left(1-f_{D}\right)$. 


\begin{tabular}{|l|l|l|l|l|l|}
\hline$T(\mathrm{~K})$ & $G_{0}$ (\# defects/atom s) & $f_{I}^{U}$ & $f_{V}^{U}$ & $S_{I}$ & $S_{V}$ \\
\hline 300 & $4.3 \times 10^{-8}$ & 0.67 & 0.46 & 2.20 & 1.63 \\
\hline 1025 & $3.3 \times 10^{-8}$ & 0.75 & 0.56 & 2.50 & 1.86 \\
\hline 2050 & $3.1 \times 10^{-8}$ & 0.66 & 0.73 & 2.17 & 2.42 \\
\hline
\end{tabular}

Table 2: Values of the parameters for the defect production rate $G_{0}$, unclustered SIA-fraction/Vfraction $f_{I}^{U}$ and $f_{V}^{U}$ and power law exponents $S_{I}$ and $S_{V}$, estimated for the temperatures $300 \mathrm{~K}, 1025$ $\mathrm{K}$ and $2050 \mathrm{~K}$, using the MD-results at the temperatures used in [51].

\subsection{Implementation}

The recrystallization model and the cluster dynamics model are solved incrementally, where the different parts of the model are solved sequentially as follows (see Figure 4):

1. Time step determination for the particular time increment $\Delta t=t_{n+1}-t_{n}$;

2. Defect evolution: calculation of the updated defect concentrations for each different representative grain, using the subincremental solution of the cluster dynamics model, between time $t_{n}$ and $t_{n+1}$;

3. Redistribution of the representative grains among the LD- and HD-sets, based on their stored defect energies $E^{V}$. When $E^{V}$ of a grain is above the threshold energy $E_{T}$, here taken equal to $5 * 10^{5} \mathrm{~J} / \mathrm{m}^{3}$, it is placed in the HD-set.Nucleated grains are initially defectfree and thus always start out in the LD-set;

4. Nucleation: the amount of new nuclei and their size are calculated; All the other grains lose a small part of their volume towards the new nuclei; Either the new nuclei are represented by a new LD-grain, or the new nuclei are merged with an existing representative grain, using the method described above;

5. Grain growth: the new grain sizes and new defect densities as a result of grain growth are calculated for each grain. Subincrements are sometimes used in this step, when grains are vanishing.

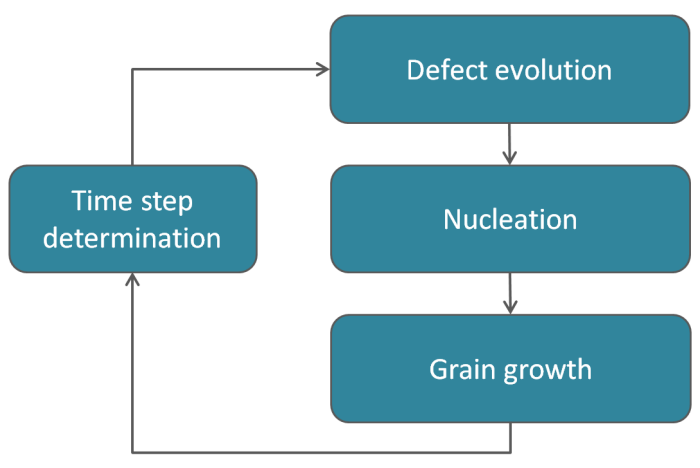

Figure 4: Solution procedure for the model of neutron-induced recrystallization.

Time step determination The size of a time step is calculated in such a way that none of the processes of defect accumulation by irradiation, grain growth and nucleation are radically changing the microstructure in a single time step. Several bounds for the time step size are calculated, for which the most stringent one applies. For grain growth, based on the volume changes $\frac{|\Delta V|}{V}$ in the previous time step $t_{n}$, the size of the new time step $\Delta t_{n+1}$ is limited such that none of the grains grow or shrink with more than (approximately) $2 \%$ :

$$
\Delta t_{n+1}<\frac{0.02 \Delta t_{n}}{\max _{i}\left(\frac{|\Delta V|_{i}}{V_{i}}\right)} .
$$


For irradiation, the time step is limited such that no grain growes more in volumetric defect energy than $10 \%$ of their grain boundary surface energy per volume. Furthermore, during nucleation, no representative grain may lose more than $3 \%$ of its total volume during the next time step (estimated, based on the volume losses during the previous time step). The time step is also not allowed to increase with more than $5 \%$ compared to the previous one. Also, for the neutron-induced recrystallization simulations, it was chosen here to not allow the time step to increase 1 hour.

When it is detected that a grain disappears during the grain growth phase, the grain growth calculations are first completed until the exact simulation time where the grain disappears, and then for the remaining time, with updated grain growth rates, to prevent an overshoot in grain growth due to a grain that has already disappeared.

A maximum is set for the number of representative recrystallized grains to limit the computation time. When nucleation occurs during a time step, and the maximum number of representative grains has been reached, then either the new grains are added to an existing representative grain (and the grain properties for grain size and defect densities are averaged based on volume), or the new grains become a new representative grain, and two existing representative grains are merged.

The two most similar representative grains based on the least-square differences of their grain surface and volumetric stored energies $E^{S}$ and $E^{V}$ are selected for merging (averaging their properties in grain size and defect densities). A representative grain that represents a large part of the total microstructural volume (here: more than $5 /$ total number of grains) is not eligible for merging.

Numerical solution For each global time increment, the set of coupled first order nonlinear stiff differential equations of the cluster dynamics model is solved with Matlab's solver ode15s, using a relative tolerance of $10^{-3}$ and requiring non-negativity. 


\section{RESULTS}

\subsection{Characterization of the nucleation parameters}

To characterize the mobility parameter $K_{m}$, the nucleation rate constant $K_{n}$ and the parameter for the reduced activation energy $K_{a}$, static recrystallization (SRX) simulations were performed with the mean-field recrystallization model and compared to experimental results of SRX-experiments performed by Lopez et al. on tungsten at several temperatures [15, 76]. EBSD measurements and hardness measurements provide information on the initial and final grain size, the initial dislocation density and the evolution of the recrystallized fraction in time [76]. Characterization based on this data, using normal distributions for the initial microstructure, resulted in a parameter set that adequately captures the effect of temperature on the time scale of the recrystallization process for all the reported temperatures, ranging from $1100^{\circ} \mathrm{C}-1250^{\circ} \mathrm{C}$, as shown in Figure 5.

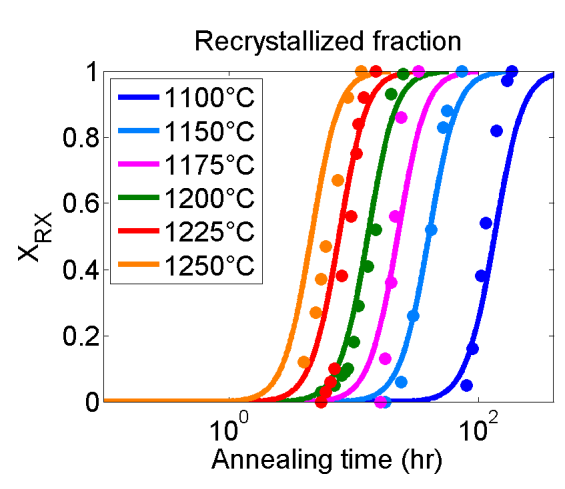

\begin{tabular}{|l|l|}
\hline Property & Value \\
\hline $\begin{array}{l}\text { Initial microstructure } \\
\text { Initial average grain size } \bar{r}_{i}\end{array}$ & $18.6 \mu \mathrm{m}[76]$ \\
$\begin{array}{l}\text { Standard deviation for } \bar{r}_{i} \\
\text { Initial average dislocation }\end{array}$ & $3.1 \mu \mathrm{m}$ \\
density $\bar{\rho}_{i}$ & $3.2 \times 10^{14} \mathrm{~m}^{-2}[76]$ \\
Standard deviation for $\bar{\rho}_{i}$ & $5.2 \times 10^{13} \mathrm{~m}^{-2}$ \\
Final microstructure & \\
Final average grain size $\bar{r}_{f}$ & $54.1 \mu \mathrm{m}[76]$ \\
$\left(\right.$ at $\left.T=1200^{\circ} \mathrm{C}\right)$ & \\
Final dislocation density $\rho_{f}$ & $1 \times 10^{9} \mathrm{~m}^{-2}[8]$ \\
\hline
\end{tabular}

Figure 5: Results of simulation of the evolution of the recrystallized volume fraction $X_{R X}$ as a function of heating time during static recrystallization, for various temperatures, compared to experimental results obtained from hardness measurements and EBSD measurements by Lopez [76] for samples that were warm-rolled to a reduction in thickness by $90 \%$. The mean-field model uses the microstructural information as listed in the table, which is based on [76].

Proper identification of the static recrystallization experiments could only be achived by adopting a different mobility for the original $\left(K_{m_{0}}\right)$ and recrystallized $\left(K_{m}\right)$ grain boundaries. In these simulations, a grain boundary is considered to be pinned when it is a boundary between two original grains (originally present in the microstructure). With this, the following parameter set is obtained: $K_{a}=1 \times 10^{8}, K_{m}=1.8 \times 10^{4}, K_{m_{0}}=25, K_{N}=3.16 \times 10^{17} \mathrm{~m}^{-2} \mathrm{~s}^{-1}$. 


\subsection{Defect evolution inside a single grain}

The defect evolution in a single grain under neutron irradiation is described with the cluster dynamics model. In Figure 6, the defect concentration distribution of the V-and SIA-clusters is shown along with the evolution of the stored energy inside a single grain, for a range of temperatures in the absence of grain growth. A maximum cluster size of 50 or 100 (for $T=1000^{\circ} \mathrm{C}$ ) is used in all simulations, to limit the computation time. For all temperatures, the stored energy increases within tens of hours to a value beyond $0.5 \mathrm{MJ} / \mathrm{m}^{3}$, which suffices for static recrystallization of a predeformed sample (e.g. 600 hours to full recrystallization, for annealing at $T=1200{ }^{\circ} \mathrm{C}$, for a warm-rolled sample reduced in thickness by $67 \%$, see [76]).

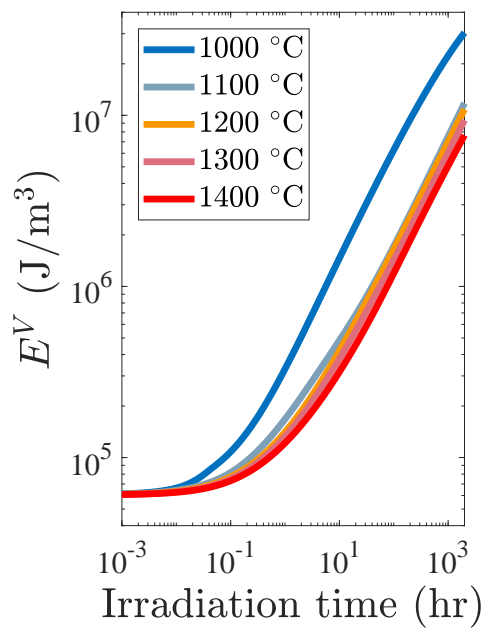

(a)

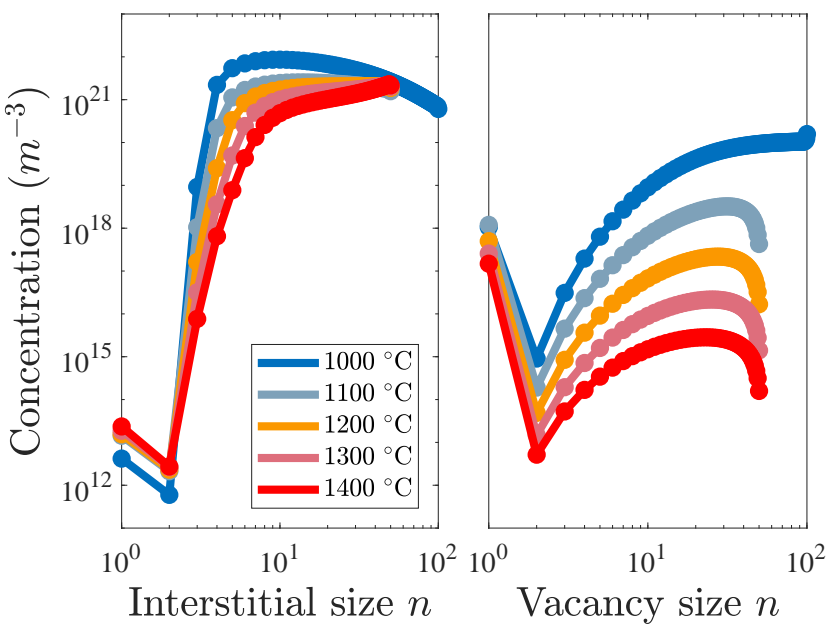

(b)

Figure 6: (a) Evolution of the volumetric stored energy inside a single grain with a radius of $3 \mu \mathrm{m}$ without recrystallization or grain growth, using $\rho_{0}=10^{13} \mathrm{~m}^{-2}$ and equilibrium concentrations for the vacancies and interstitials, using $N=100$ at $T=1000{ }^{\circ} \mathrm{C}$ and $N=50$ at the other temperatures; (b) temperature dependent defect distributions for interstitial and vacancy clusters of different sizes after irradiation for $100 \mathrm{hrs}$, without recrystallization or grain growth.

The temperature dependence of the defect concentrations originates from the increased mobility of the mobile defects at a higher temperature and from a different defect production term (decreasing total production, more production of isolated SIAs and less production of isolated vacancies), see section 2.3.4. Combined, the different mobility and cluster distributions affect the rates of the following processes for mobile defects:

- annihilation at dislocation and grain boundary sinks;

- annihilation by recombination with mobile defects of the opposite type (cluster sinks);

- annihilation at defect clusters of the opposite type;

- formation of larger clusters by absorption at clusters of the same type;

- emission from clusters of the same type. 


\subsection{Neutron-induced recrystallization}

The effect of temperature on the balance between the neutron-induced damage accumulation and recovery by grain growth and recrystallization, as well as on the resulting microstructural evolution, is investigated next.

\subsubsection{Temperature dependent microstructural evolution}

Simulations of the microstructure evolution under irradiation at various temperatures ranging from $T=1000{ }^{\circ} \mathrm{C}$ to $T=1400{ }^{\circ} \mathrm{C}$ have been performed, for an initial microstructure consisting of 150 representative grains with a normal size distribution, with number average $\overline{r_{0}}=3 \mu \mathrm{m}$ and a volume average of $2.8 \mu \mathrm{m}$ and standard deviation $\sigma_{\bar{r}_{0}}=0.5 \mu \mathrm{m}, \rho_{0}=10^{13} \mathrm{~m}^{-2}$ and equilibrium concentrations ${ }^{2}$ for the defect clusters. The maximum cluster sizes of before, $N=50$ and $N=100$, are used.

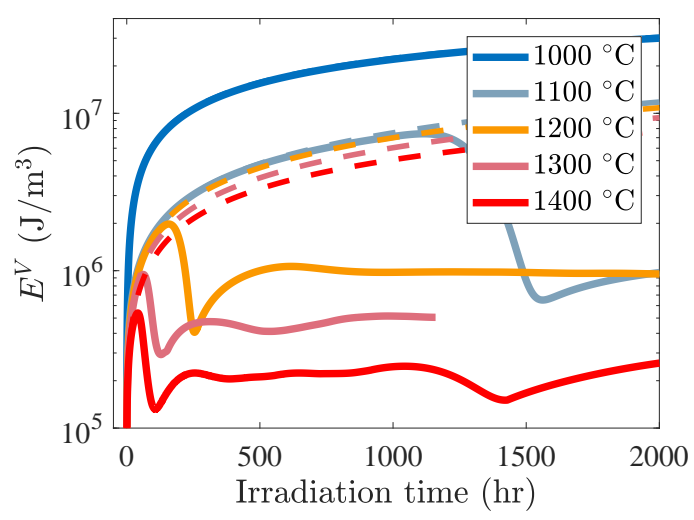

(a)

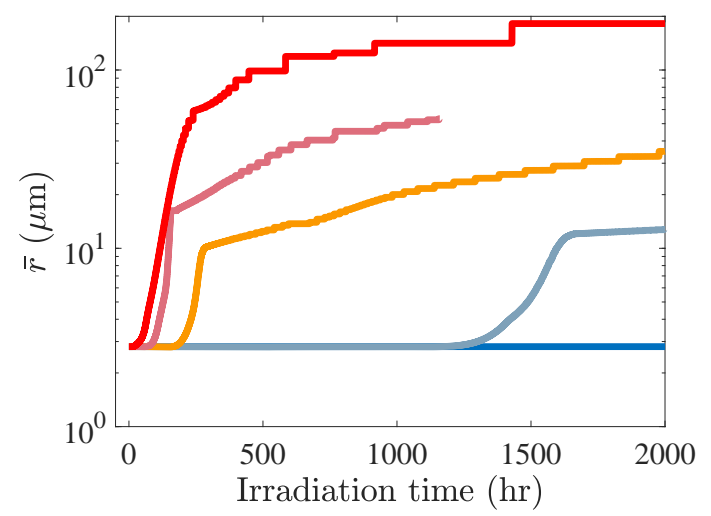

(b)

Figure 7: (a) Temperature dependence of the volume average of the stored volumetric defect energy $E^{V}$ (solid lines), compared to the evolution of $E^{V}$ for a single grain of $3 \mu \mathrm{m}$, (dashed lines, without grain growth), see also Figure $6 \mathrm{a}$ and (b) temperature dependence of the evolution of the (volumetric) average grain size $\bar{r}$.

Figure 7 shows the evolution of the main microstructural properties (defect energy density and grain size) under these conditions. A strong temperature dependence of the microstructure can be observed: the difference in the volumetric stored energy $E^{V}$ at different temperatures reaches 2 orders of magnitude. At $T=1400{ }^{\circ} \mathrm{C}$, the mobility is much higher than at e.g. $T=1100$ ${ }^{\circ} \mathrm{C}$, i.e. significantly more recovery occurs, in the form of defect sweeping during grain growth and nucleation of new grains, as becomes clear from the comparison with the evolution of $E^{V}$ for a single grain without grain growth. However, even at $T=1100{ }^{\circ} \mathrm{C}$, significant recovery still takes place too, by nucleation and grain growth, since $E^{V}$ stays lower in Figure 7 than for the single grain simulation. For $T=1000{ }^{\circ} \mathrm{C}, E^{V}$ does notdeviate significantly from the single grain behaviour (Figure 6a) within the first 2500 hours.

Damage and recovery Undulations are observed for the volumetric stored energy $E^{V}$ in Figure 7a. To identify the origin of these undulations, the competition between the damage growth rate $\dot{E}_{I R R}^{V}$ (due to production and evolution of displacement defects inside the grains, given by the CD-model) and the recovery rate $-\dot{E}_{R X}^{V}$ by grain growth and nucleation (the RXmodel) is assessed. Figure 8a reveals how these rates influence $E^{V}$ for a temperature of $T=1400$ ${ }^{\circ} \mathrm{C}$ (at other temperatures, a similar behaviour is found).

The irradiation damage growth rate is the highest at the start of the simulation, when the grains have not built up any displacement damage yet. Thereafter, the irradiation damage rate decreases monotonically. The recovery rate has a broad peak at roughly 40-100 hours. The high recovery rate during the process of recrystallization leads to a large drop in the volumetric stored

\footnotetext{
${ }^{2}$ The equilibrium defect concentrations were calculated based on the defect formation energies, e.g. $C^{e q}\left(I_{n}\right)=$ $\frac{1}{V} \exp \left(E^{f}\left(I_{n}\right) / k_{B} T\right)$.
} 


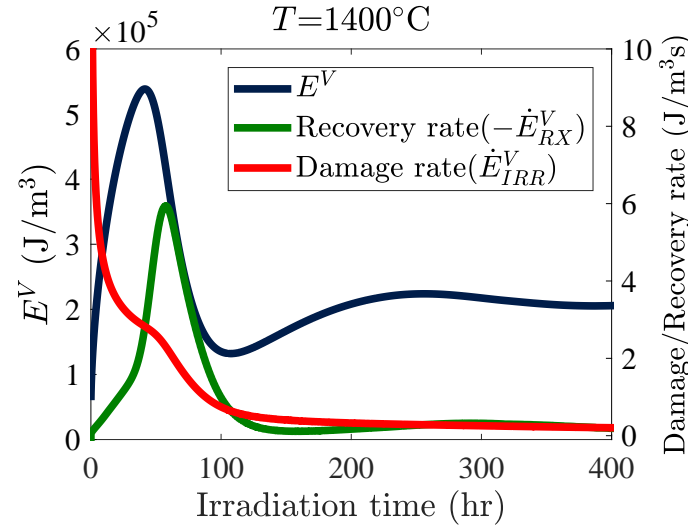

(a)

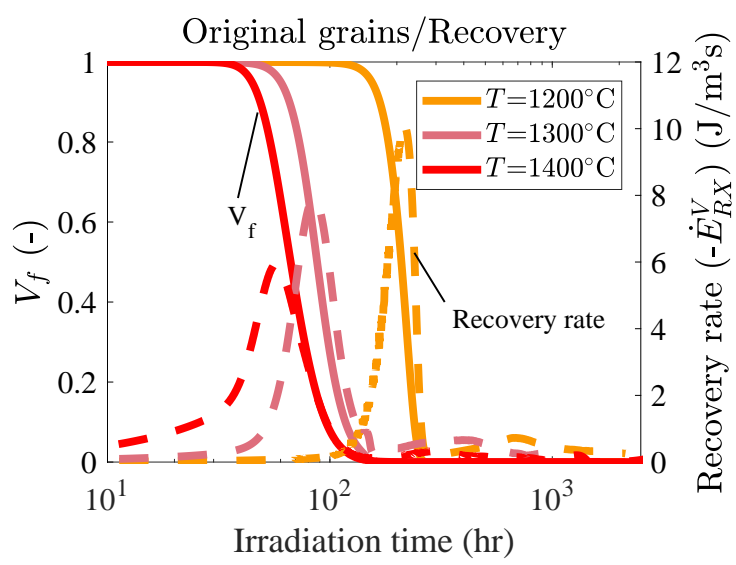

(b)

Figure 8: (a) Evolution of $E^{V}$ at $T=1400{ }^{\circ} \mathrm{C}$ due to the average recovery rate $\left(-\dot{E}_{R X}^{V}\right)$ during grain growth and nucleation and due to the average damage increase rate $\dot{E}_{I R R}^{V}$ during the production and evolution of the displacement defects inside each grain; (b) the evolution of the average recovery rate $\left(-\dot{E}_{R X}^{V}\right)$ is compared to the remaining volume fraction of the original grains as a function of irradiation time, for temperatures $T=1200{ }^{\circ} \mathrm{C}$ up to $T=1400{ }^{\circ} \mathrm{C}$.

energy of the microstructure $E^{V}$. Figure $8 \mathrm{~b}$ shows the same recovery rate, for temperatures $T=1200{ }^{\circ} \mathrm{C}$ up to $T=1400{ }^{\circ} \mathrm{C}$, in comparison to the volume fraction of the grains that made up the original microstructure. From Figure $8 \mathrm{~b}$ it becomes clear that the peak in the recovery rate (and thus the drop in $E^{V}$ ) is caused by the shrinkage and subsequent loss of a large amount of grains. 


\subsubsection{Microstructural evolution at $1300{ }^{\circ} \mathrm{C}$}

The mechanisms governing the microstructural evolution are discussed next using the results for an irradiation temperature of $1300{ }^{\circ} \mathrm{C}$. In Figure 9, the resulting evolution of grain size and volumetric defect energy of the grains during irradiation at this temperature are depicted for selected grains, both from the original grain set and freshly nucleated grains. In Appendix C, similar graphs are shown for the other simulated temperatures. The grains are irradiated and

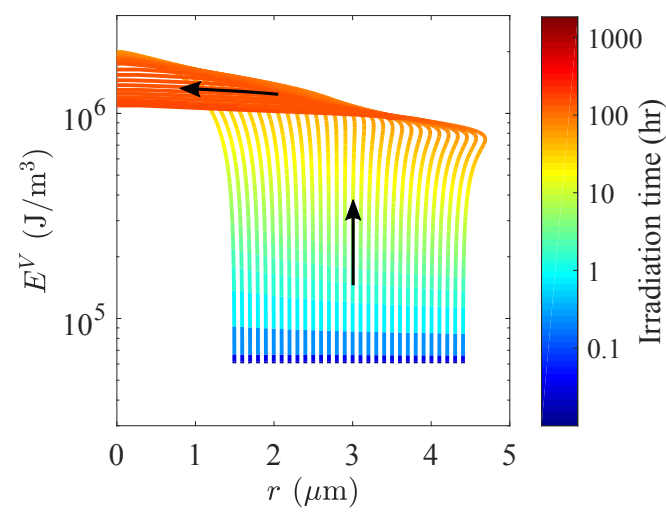

(a)

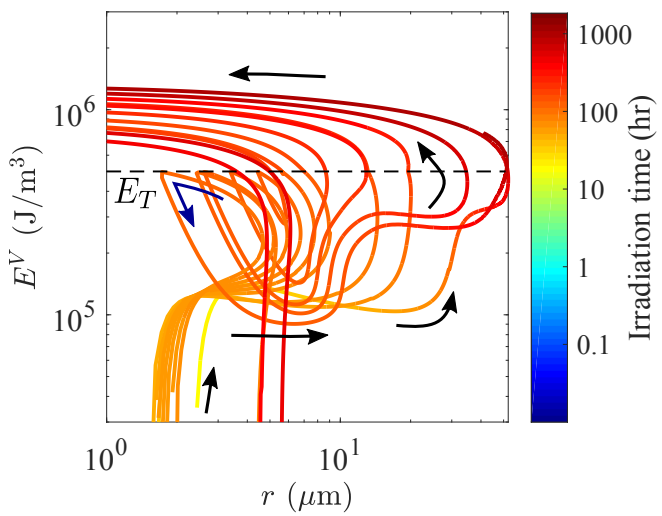

(b)

Figure 9: Temporal evolution of the grain radius $r$ and the volumetric stored defect energy $E^{V}$ as a function of the irradiation time at $T=1300{ }^{\circ} \mathrm{C}$ for a selection of representative grains from (a) the original grain set and (b) the nucleated grains. The black arrows denote the typical temporal evolution in grain size and volumetric stored energy for individual grains. The blue arrow in (b) denotes the consequence of reaching the threshold energy on the $\left(r, E^{V}\right)$-path of some of the nucleated grains.

accumulate defect energy. The smallest grains vanish first, because the grain growth is driven by the grain boundary energy (i.e. grain sizes), while the larger original grains first grow before they finally shrink as well. After around 300 hours of irradiation, all the original grains have vanished. The original grains reach a maximum size not exceeding $r=5 \mu \mathrm{m}$. The new (nucleated) grains grow to much larger sizes than the original grains. The growth of the new grains occurs fast, due to the high driving force and a high grain boundary mobility. While growing, a lot of defect-free volume is constantly added to the grain volume, which slows down the accumulation of defect energy in those grains. Once the grains are large, they accumulate damage much faster, as the grain boundary, which acts as a sink, cannot be reached easily by most mobile defects. As a result, the new grains will not grow anymore, but shrink rapidly instead. While shrinking, $E^{V}$ keeps increasing, thereby accelerating the process of shrinkage. This process is continuously repeated until the nucleation rate dropped to a level at which hardly any new grains are further nucleated. The nucleation rate decreases a lot as the original grains vanish, because with their extinction the amount of grain boundary available for heterogeneous nucleation decreases. The alternating shrinkage and growth of grains explains the undulations observed in Figure 7. This is particularly clear for the minimum in $E^{V}$ (Figure 6a) at an irradiation time of approximately 50 hours, the moment where many of the original grains are shrinking until they vanish (Figure $7 \mathrm{a}$ and $8 \mathrm{~b})$.

The nucleated grains are incorporated in the low-defect density HEM (the LD-HEM) and are placed in the HD-HEM upon reaching threshold energy $E_{T}$. Figure $9 \mathrm{~b}$ points out that for some of the nucleated grains the amount of growth/shrinkage changes upon changing the HEM. It may happen that grains that were initially shrinking, start growing again. This effect shows the need to use multiple HEMs in the mean-field model, for which the use of more than two HEMs may result in a more realistic description of grain growth. This will be explored in a future extension of the model. 


\subsubsection{Nucleation}

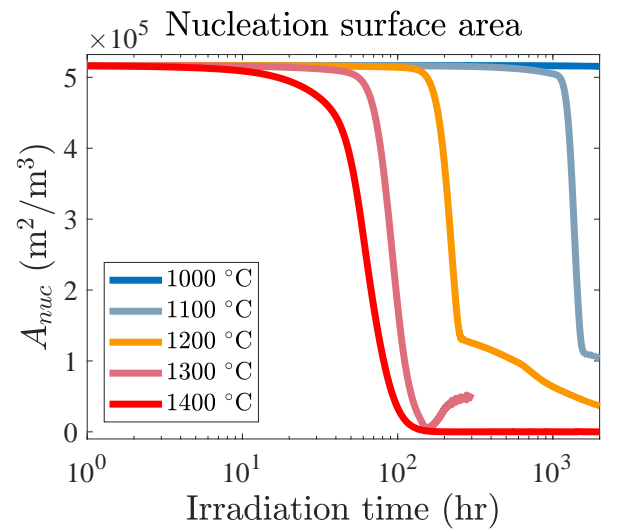

(a)

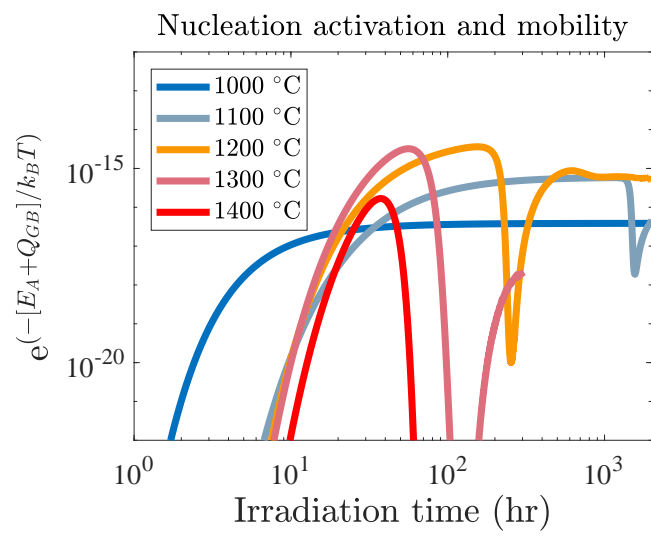

(c)

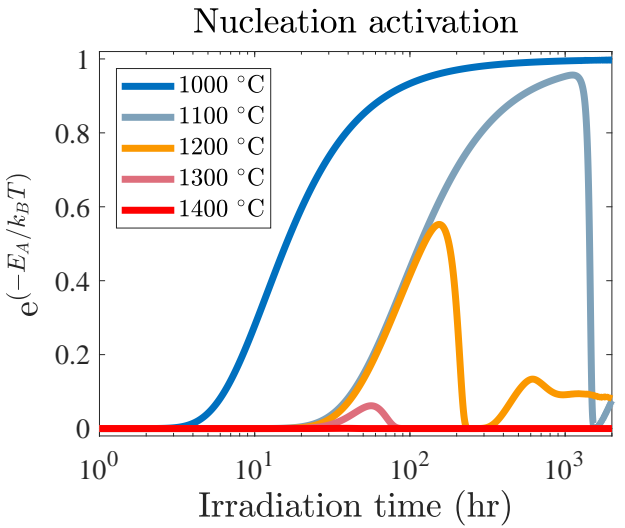

(b)

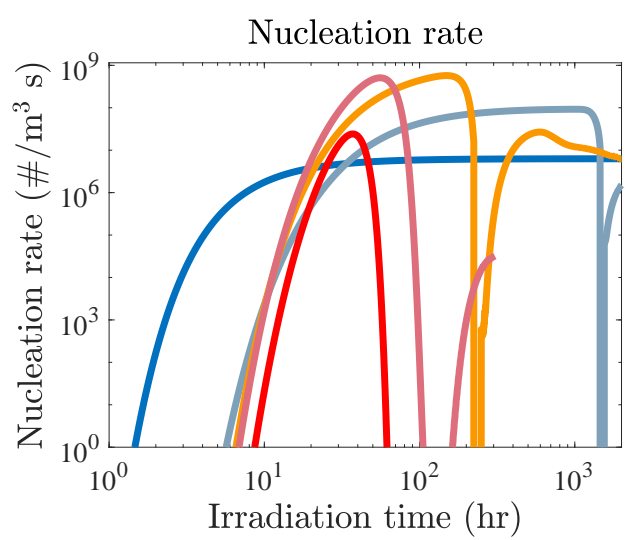

(d)

Figure 10: Temperature dependence of the nucleation process. The contributions of (a) the nucleation surface area, (b) the nucleation activation term and (c) the grain boundary mobility to the resulting nucleation rate $(\mathrm{d})$ are shown.

In Figure 10, the temperature dependence of the nucleation behaviour is shown. During the first 10 hours of irradiation, most nucleation takes place for an irradiation temperature of $1000{ }^{\circ} \mathrm{C}$ (Figure 10a), even though the grain boundary mobility is quite low at this temperature, because the activation energy is already low (Figure 10b). The nucleation rate is higher for the other temperatures (Figure 10d). For $T=1400{ }^{\circ} \mathrm{C}$, the nucleation rate decreases quickly, because the nucleation surface area decreases when the original grains vanish (Figure 8b), and the newly formed grains, which reside in the low-defect density $\operatorname{HEM}\left(E_{\text {grain }}<E_{T}\right)$, do not contribute to the nucleation surface area. The nucleation surface area also decreases for the temperatures of $T=1200{ }^{\circ} \mathrm{C}$ and $T=1300{ }^{\circ} \mathrm{C}$. At these temperatures, the nucleated grains accumulate more damage, through which they get shifted to the high-defect HEM after some time, thereby again increasing the surface area at which nucleation can take place. By comparing Figure 10b and $10 \mathrm{c}$, it is obvious that the activation energy and grain boundary mobility balance out for each temperature. The nucleation rate is the highest for temperatures $T=1100{ }^{\circ} \mathrm{C}$ up to $T=1300$ ${ }^{\circ} \mathrm{C}$ (depending on the time scale). At $1000{ }^{\circ} \mathrm{C}$ the activation energy is low but the mobility is insufficient and at $1400{ }^{\circ} \mathrm{C}$ this situation is reversed. From Figure 10b, it can also be seen that for $T=1000{ }^{\circ} \mathrm{C}$ a further decrease of the activation energy does not result in a further increase of the nucleation rate, as the exponential function already approximates 1 . At temperatures of $T=1100{ }^{\circ} \mathrm{C}$ and $T=1200{ }^{\circ} \mathrm{C}$, the nucleation rate is temporarily zero (around 1000 hours and from roughly 220-250 hours), because the activation energy is temporarily much higher, and therefore no stable nucleus is formed. 


\section{Conclusions and discussion}

For the prediction of the lifetime of the divertor in an operational fusion reactor, and the study of the physical processes that are limiting this lifetime, it is crucial to predict the evolution of the thermomechanical properties. These evolving properties are determined by the underlying microstructural evolution. Here a model framework was developed capturing the combined effects of a high temperature and (continuous) neutron-induced displacement defect load on the microstructure, using a multi-scale approach. It was shown that the model allows to assess the competition between the various processes for damage and recovery, including the mobility of the grain boundaries, and the mobility of the defects (affecting the time at which sinks are reached and its effect on the evolution of the microstructure), the nucleation rate, the defect distribution and the resulting rate at which the original microstructure vanishes. Since most of the mentioned damage/recovery effects are strongly temperature dependent, the volumetric stored defect energy also exhibits a strong temperature dependence in the range in which the model was tested, $T=1000-1400{ }^{\circ} \mathrm{C}$.

With the model, it was also explored, for small maximum sizes of the defect clusters, which grains of the original microstructure are vanishing first under the influence of nucleation and grain growth, how the sizes of the newly nucleated grains evolve and whether they are replaced again by new nucleating grains. The net result is a competition between grain boundary mobility, defect accumulation (which depends on the grain size and temperature) in relation to the grain boundary surface energy (i.e. grain size), activity of the defect sinks and the nucleation rate. To study the competing effects in detail, trajectory plots of the temporal evolution of the grain size and volumetric defect density for the individual grains are reported. It was found, within the limitations of the modelling conditions, that nucleated grains often grow to sizes of at least several tens of $\mu \mathrm{m}$. Once these grains have become large, they accumulate a larger defect energy density, upon which they shrink again. During this shrinking phase, the defect energy density remains high for the set of parameters considered here. Moreover, for the given parameter set, effects of recrystallization and grain growth are clearly active, starting from irradiation temperatures of $1100{ }^{\circ} \mathrm{C}$, and the original microstructure was shown to be completely replaced within several hundreds of hours: within 1500 hours for irradiation at $1100{ }^{\circ} \mathrm{C}$ up to within 200 hours for irradiation at $1400{ }^{\circ} \mathrm{C}$.

Also in this work for the first time a temperature dependent scaling law was used in cluster dynamics simulations as a source term for clustered production of displacement defects.

The results of the presented framework can be further improved by: (1) increasing the maximum cluster size (beyond 50 or 100 that was used here). Larger cluster sizes are needed for a realistic description of the evolution of lattice damage, for which the unfaulting of prismatic dislocation loops can also be included. Test simulations (not shown) revealed that especially at $T=1000{ }^{\circ} \mathrm{C}$ it is necessary to use a high maximum cluster size; (2) the use of more than two homogeneous equivalent media in the mean-field recrystallization model. This would improve the grain growth behaviour and also it would make the nucleation rate less sensitive to the theshold energy (which currently regulates the switch of nucleated grains towards the high defect density medium) (3) incorporating the influence of stress on the recrystallization process into account and the effect of recrystallization on the stress state in the material;

Furthermore, in the current model, nucleation and grain growth are thermally-activated. It is possible that irradiation alone may be responsible for (some) grain growth and nucleation. In that case, recrystallization could already take place at low(er) temperatures. Moreover, currently a necklace-type nucleation was assumed. However, for heavy irradiation, it is expected that nucleation may also take place in the grain interior in addition to at the grain boundary. In the current model, the SIA-clusters are assumed to be of a 2D-shape and the V-clusters are assumed to be of a 3D-shape. MD-simulations indicate however that SIA-clusters can take 2D or 3D shapes [51]. No impurities or neutron-induced transmutations were considered here.

The model can be validated by performing irradiation experiments on tungsten at high tem- 
peratures and by measuring the temporal evolution of the grain size and dislocation density and/or the concentrations of vacancies and self-interstitial (cluster) defects. Self-ions and protons are the most suitable particles to simulate irradiation in this case, since hereby no additional impurities are introduced into the material.

In the future, this type of modelling can be used to examine the effect of heat treatments for interim recovery of the divertor component, which enables a route towards extending the component's lifetime. If the optimum microstructural pathway corresponding to the desired thermomechanical evolution for the divertor is known, then the heat treatments can be used to optimally modify the grain sizes and defect (energy) densities in the microstructure.

\section{Acknowledgements}

The authors would like to thank prof.dr. N.J. Lopes Cardozo and dr.ir. J.H.M. ten Thije Boonkkamp for the fruitful discussions and valuable suggestions.

\section{Declarations of interest:}

None. 


\section{A Entropy of point defect clusters}

The point defect clusters contribute to the entropy of mixing of the system. As long as the defect density can be called dilute, the sum of all the individual contributions of each defect type to the mixing entropy is given by [80].

$$
S=k_{B} \ln \Omega,
$$

where $S$ is the entropy $\left(\mathrm{J} / \mathrm{m}^{3}\right)$ and $\Omega$ is the number of possible configurations of the defects in the material, with [80]:

$$
\begin{aligned}
\ln \Omega=\sum_{m} \ln \Omega_{m}= & \sum_{m}\left[\ln \frac{m^{N(m)}\left(\frac{N_{S}}{m}\right) !}{\left(\frac{N_{S}}{m}-N(m)\right) !(N(m)) !}\right] \\
= & \sum_{m}\left[N(m) \ln m+\frac{N_{S}}{m} \ln \left(\frac{N_{S}}{m}\right)\right. \\
& -\left[\frac{N_{S}}{m}-N(m)\right] \ln \left[\frac{N_{S}}{m}-N(m)\right] \\
& -N(m) \ln [N(m)]]
\end{aligned}
$$

Here $m$ denotes the number of point defects in the cluster, $N_{S}$ is the number of lattice sites that is availabe in a unit volume. If the unit volume is $1 \mathrm{~m}^{3}$, then $N_{S}=1 / V_{a t}$ and if the concentrations are in atomic units, then $N_{S}=1$. In $(21), N(m)$ is the defect distribution.

\section{B Cluster evolution details}

The cluster dynamics model is given by the following set of equations, adapted from [8]:

$$
\begin{aligned}
& \frac{d C_{I}}{d t}=G_{I}+2 \alpha_{2}^{-} C_{I_{2}}+\sum_{n=3}^{N_{I}} \alpha_{n}^{-} C_{I_{n}}+\beta_{3}^{-} C_{I_{3}}+\left(k_{I_{2}+V}^{+}+k_{V_{1}+I_{2}}^{+}\right) C_{I_{2}} C_{V}-2 \alpha_{1}^{+} C_{I}^{2} \\
& -\left(\alpha_{2}^{+}+\beta_{1}^{+}\right) C_{I} C_{I_{2}}-\sum_{n=3}^{N_{I}-1} \alpha_{n}^{+} C_{I} C_{I_{n}}-k_{I+V}^{+}\left(C_{I} C_{V}-C_{I}^{e q} C_{V}^{e q}\right) \\
& -\sum_{n=2}^{N_{V}} k_{V_{n}+I}^{+} C_{I} C_{V_{n}}-k_{I_{1}-V}^{-} C_{I}-\left(k_{D+I}^{+}+k_{S+I}^{+}\right) C_{I} \\
& \frac{d C_{I_{2}}}{d t}=G_{I_{2}}+\alpha_{1}^{+} C_{I}^{2}+k_{I_{3}+V}^{+} C_{I_{3}} C_{V}+\alpha_{3}^{-} C_{I_{3}}+\beta_{3}^{-} C_{I_{3}}+2 \beta_{4}^{-} C_{I_{4}}+\sum_{n=5}^{N_{I}} \beta_{n}^{-} C_{I_{n}} \\
& +k_{I-V}^{-} C_{I}-\alpha_{2}^{-} C_{I_{2}}-\left(\beta_{1}^{+}+\alpha_{2}^{+}\right) C_{I_{2}} C_{I}-2 \beta_{2}^{+} C_{I_{2}}^{2}-k_{I_{2}-V}^{-} C_{I_{2}}-\sum_{n=3}^{N_{I}-2} \beta_{n}^{+} C_{I_{2}} C_{I_{n}} \\
& -\left(k_{I_{2}+V}^{+}+k_{V_{1}+I_{2}}^{+}\right) C_{V} C_{I_{2}}-\sum_{n=2}^{N_{V}} k_{V_{n}+I_{2}}^{+} C_{V_{n}} C_{I_{2}}-\left(k_{D+I_{2}}^{+}+k_{S+I_{2}}^{+}\right) C_{I_{2}} \\
& {\frac{d C_{I_{n}}}{d t}{ }_{3 \leq n \leq N_{I}-2}}=G_{I_{n}}+\alpha_{n+1}^{-} C_{I_{n+1}}+\beta_{n+2}^{-} C_{I_{n+2}}+\alpha_{n-1}^{+} C_{I} C_{I_{n-1}}+\beta_{n-2}^{+} C_{I_{2}} C_{I_{n-2}} \\
& +k_{I_{n-1}-V}^{-} C_{I_{n-1}}+k_{I_{n+1}+V}^{+} C_{V} C_{I_{n+1}}-\alpha_{n}^{+} C_{I} C_{I_{n}}-\alpha_{n}^{-} C_{I_{n}} \\
& -\beta_{n}^{+} C_{I_{2}} C_{I_{n}}-\beta_{n}^{-} C_{I_{n}}-k_{I_{n}+V}^{+} C_{V} C_{I_{n}}-k_{I_{n}-V}^{-} C_{I_{n}} \\
& \frac{d C_{I_{N_{I}-1}}}{d t}=G_{I_{N_{I}-1}}+\alpha_{N_{I}}^{-} C_{I_{N_{I}}}+\alpha_{N_{I}-2}^{+} C_{I} C_{I_{N_{I}-2}}+\beta_{N_{I}-3}^{+} C_{I_{2}} C_{I_{N_{I}-3}} \\
& +k_{I_{N_{I}-2}-V}^{-} C_{I_{N_{I}-2}}+k_{I_{N_{I}}+V}^{+} C_{V} C_{I_{N_{I}}}-\beta_{N_{I}-1}^{-} C_{I_{N_{I}-1}}-\alpha_{N_{I}-1}^{+} C_{I} C_{I_{N_{I}-1}} \\
& -\alpha_{N_{I}-1}^{-} C_{I_{N_{I}-1}}-k_{I_{N_{I}-1}+V}^{+} C_{V} C_{I_{N_{I}-1}}-k_{I_{N_{I}-1}-V}^{-} C_{I_{N_{I}-1}}
\end{aligned}
$$




$$
\begin{aligned}
& \frac{d C_{I_{N_{I}}}}{d t}=G_{I_{N_{I}}}+\alpha_{N_{I}-1}^{+} C_{I} C_{I_{N_{I}-1}}+\beta_{N_{I}-2}^{+} C_{I_{2}} C_{I_{N_{I}-2}} \\
& +k_{I_{N_{I}-1}-V}^{-} C_{I_{N_{I}-1}}-\alpha_{N_{I}}^{-} C_{I_{N_{I}}}-\beta_{N_{I}}^{-} C_{I_{N_{I}}}-k_{I_{N_{I}}+V}^{+} C_{V} C_{I_{N_{I}}} \\
& \frac{d C_{V}}{d t}=G_{V}+2 \gamma_{2}^{-} C_{V_{2}}+k_{V_{2}+I}^{+} C_{V_{2}} C_{I}+k_{V_{3}+I_{2}}^{+} C_{V_{3}} C_{I_{2}}+\sum_{n=3}^{N_{V}} \gamma_{n}^{-} C_{V_{n}} \\
& +\sum_{n=1}^{N_{I}-1} k_{I_{n}-V}^{-} C_{I_{n}}-k_{I+V}^{+}\left(C_{I} C_{V}-C_{I}^{e q} C_{V}^{e q}\right)-2 \gamma_{1}^{+} C_{V}^{2}-\sum_{n=2}^{N_{V}-1} \gamma_{n}^{+} C_{V} C_{V_{n}} \\
& -\left(k_{V_{1}+I_{2}}^{+}+k_{I_{2}+V}^{+}\right) C_{I_{2}} C_{V}-\sum_{n=3}^{N_{I}} k_{I_{n}+V}^{+} C_{V} C_{I_{n}}-\left(k_{D+V}^{+}+k_{S+I}^{+}\right) C_{V} \\
& \frac{d C_{V_{n}}}{d t}{ }_{2 \leq n \leq N_{V}-2}=G_{V_{n}}+k_{V_{n+1}+I}^{+} C_{I} C_{V_{n+1}}+\gamma_{n+1}^{-} C_{V_{n+1}}+k_{V_{n+2}+I_{2}}^{+} C_{I_{2}} C_{V_{n+2}} \\
& +\gamma_{n-1}^{+} C_{V} C_{V_{n-1}}-k_{V_{n}+I}^{+} C_{I} C_{V_{n}}-\gamma_{n}^{-} C_{V_{n}}-\gamma_{n}^{+} C_{V} C_{V_{n}}-k_{V_{n}+I_{2}}^{+} C_{V_{n}} C_{I_{2}} \\
& \frac{d C_{V_{N_{V}-1}}}{d t}=G_{N_{N_{V}-1}}+k_{V_{N_{V}}+I}^{+} C_{I} C_{V_{N_{V}}}+\gamma_{N_{V}}^{-} C_{V_{N_{V}}} \\
& +\gamma_{N_{V}-2}^{+} C_{V} C_{V_{N_{V}-2}}-k_{V_{N_{V}-1}+1}^{+} C_{I} C_{V_{N_{V}-1}}-\gamma_{N_{V}-1}^{-} C_{V_{N_{V}-1}} \\
& -\gamma_{N_{V}-1}^{+} C_{V} C_{V_{N_{V}-1}}-k_{V_{N_{V}-1}+I_{2}}^{+} C_{V_{N_{V}-1}} C_{I_{2}} \\
& \frac{d C_{V_{N_{V}}}}{d t}=G_{N_{N_{V}}}+\gamma_{N_{V}-1}^{+} C_{V} C_{V_{N_{V}-1}}-k_{V_{N_{V}}+I}^{+} C_{I} C_{V_{N_{V}}}-\gamma_{N_{V}}^{-} C_{V_{N_{V}}}-k_{V_{N_{V}}+I_{2}}^{+} C_{V_{N_{V}}} C_{I_{2}} \text {, }
\end{aligned}
$$

where all the rate coefficients, binding energies and diffusion coefficients entering the equations are listed in Table 4 and the parameter values are listed in Table 7. 
Sink strength sum for GB-sink strength calculation (based on [Bullough1980])

$$
\begin{aligned}
& \left(S_{I}^{s k}\right)^{2}=\frac{1}{D_{I}}\left[\sum_{n=1}^{N_{I}-1} \alpha_{n}^{+} C_{I_{n}}+\sum_{n=1}^{N_{V}} k_{V_{n}+I}^{+} C_{V_{n}}\right]+\rho_{D} Z_{D}^{I} \\
& \left(S_{I_{2}}^{s k}\right)^{2}=\frac{1}{D_{I_{2}}}\left[\sum_{n=1}^{N_{I}-2} \beta_{n}^{+} C_{I_{n}}+\sum_{n=1}^{N_{V}} k_{V_{n}+I_{2}}^{+} C_{V_{n}}\right]+\rho_{D} Z_{D}^{I_{2}} \\
& \left(S_{V}^{s k}\right)^{2}=\frac{1}{D_{V}}\left[\sum_{n=1}^{N_{V}-1} \gamma_{n}^{+} C_{V_{n}}+\sum_{n=1}^{N_{I}} k_{I_{n}+V}^{+} C_{I_{n}}\right]+\rho_{D} Z_{D}^{V}
\end{aligned}
$$

Sink strength coefficient for GB-sink strength calculation (based on [Bullough1980])

$$
\begin{aligned}
& \left(S_{I}^{s k}\right)^{2}=\frac{1}{D_{I}}\left[2 \alpha_{1}^{+} C_{I_{1}}+\sum_{n=2}^{N_{I}-1} \alpha_{n}^{+} C_{I_{n}}+\sum_{n=1}^{N_{V}} k_{V_{n}+I}^{+} C_{V_{n}}\right]+\rho_{D} Z_{D}^{I} \\
& \left(S_{I_{2}}^{s k}\right)^{2}=\frac{1}{D_{I_{2}}}\left[\beta_{1}^{+} C_{I_{1}}+2 \beta_{2}^{+} C_{I_{2}}+\sum_{n=3}^{N_{I}-2} \beta_{n}^{+} C_{I_{n}}+\sum_{n=1}^{N_{V}} k_{V_{n}+I_{2}}^{+} C_{V_{n}}\right]+\rho_{D} Z_{D}^{I_{2}} \\
& \left(S_{V}^{s k}\right)^{2}=\frac{1}{D_{V}}\left[2 \gamma_{1}^{+} C_{V_{1}}+\sum_{n=2}^{N_{V}-1} \gamma_{n}^{+} C_{V_{n}}+\sum_{n=1}^{N_{I}} k_{I_{n}+V}^{+} C_{I_{n}}\right]+\rho_{D} Z_{D}^{V}
\end{aligned}
$$

Binding energy (using the capillarity approximation)

$$
\begin{array}{ll}
E_{I_{n}}^{f} & =E_{I_{n-1}}^{f}+E_{I}^{f}-E_{I_{n}-I}^{b} \text { (by definition) } \\
E_{I_{n}-I}^{b} & =E_{I}^{f}+\frac{E_{I_{2}}^{b}-E_{I}^{f}}{2^{2 / 3}-1}\left[n^{2 / 3}-(n-1)^{2 / 3}\right] \\
E_{I_{n}-I_{2}}^{b} & =2 E_{I}^{f}-E_{I_{2}}^{b}+\frac{E_{I_{2}}^{b}-E_{I}^{f}}{2^{2 / 3}-1}\left[(n+1)^{2 / 3}-(n-1)^{2 / 3}\right] \\
E_{I_{n}-V}^{b} & =E_{V}^{f}+\frac{E_{I}^{f}-E_{I_{2}}^{b}}{2^{2 / 3}-n^{2}}\left[n^{2 / 3}-(n-1)^{2 / 3}\right] \\
E_{V_{n}-V}^{b} & =E_{V}^{f}+\frac{E_{V_{2}}^{b}-E_{V}^{f}}{2^{2 / 3}-1}\left[n^{2 / 3}-(n-1)^{2 / 3}\right]
\end{array}
$$

\section{Diffusion coefficients}

$$
\begin{aligned}
D_{I} & =D_{I_{0}} \exp \left(-E_{I}^{m} / k_{B} T\right) \\
D_{I_{2}} & =D_{I_{20}} \exp \left(-E_{I_{2}}^{m} / k_{B} T\right) \\
D_{V} & =D_{V_{0}} \exp \left(-E_{V}^{m} / k_{B} T\right)
\end{aligned}
$$

Dislocation bias factor

$$
\begin{aligned}
Z_{I_{n}}^{I} & =Z_{D}^{I} \max \left[\frac{2 \pi}{\ln \left(8 r_{I_{n}} / r_{p}\right)}, 1\right] \\
Z_{I_{n}}^{I_{2}} & =Z_{D}^{I_{2}} \max \left[\frac{2 \pi}{\ln \left(8 r_{I_{n}} / r_{p}\right)}, 1\right] \\
Z_{I_{n}}^{V} & =Z_{D}^{V} \max \left[\frac{2 \pi}{\ln \left(8 r_{I_{n}} / r_{p}\right)}, 1\right]
\end{aligned}
$$

Table 6: Expressions for the grain boundary sink strength, the formation energies and binding energies of the defect clusters, the diffusion coefficients of the mobile defects and the bias factors for the interaction between mobile defects and self-interstitial clusters. For the simulations, $r_{p}=2 b$ is assumed [8]. 


\begin{tabular}{|c|c|c|c|}
\hline Parameter & Unit & Value & Description \\
\hline$a_{0}$ & $\mathrm{~nm}$ & 0.31652 & Lattice parameter \\
\hline$\gamma_{b}$ & $\mathrm{~J} / \mathrm{m}^{2}$ & 0.869 & GB-surface energy \\
\hline$E_{I}^{f}$ & $\mathrm{eV}$ & 9.466 & Formation energy SIA \\
\hline$E_{V}^{f}$ & $\mathrm{eV}$ & 3.80 & Formation energy vacancy \\
\hline$E_{I_{2}}^{b}$ & $\mathrm{eV}$ & 2.12 & Binding energy SIA-SIA \\
\hline$E_{V_{2}}^{f}$ & $\mathrm{eV}$ & 0.6559 & Binding energy V-V \\
\hline$D_{I_{0}}$ & $\mathrm{~m}^{2} / \mathrm{s}$ & $8.77 \times 10^{-8}$ & SIA-diffusivity in tungsten \\
\hline$D_{I_{20}}$ & $\mathrm{~m}^{2} / \mathrm{s}$ & $7.02 \times 10^{-8}$ & Double SIA-diffusivity in tungsten \\
\hline$D_{V_{0}}$ & $\mathrm{~m}^{2} / \mathrm{s}$ & $177 \times 10^{-8}$ & Vacancy diffusivity in tungsten \\
\hline$E_{I}^{m}$ & $\mathrm{eV}$ & 0.013 & SIA migration energy \\
\hline$E_{I_{2}}^{m}$ & $\mathrm{eV}$ & 0.024 & $I_{2}$ migration energy \\
\hline$E_{V}^{m}$ & $\mathrm{eV}$ & 1.66 & Vacancy migration energy \\
\hline$Z_{D}^{I}$ & - & 1.2 & SIA-dislocation bias \\
\hline$Z_{D}^{I_{2}}$ & - & 1.2 & Double SIA-dislocation bias \\
\hline$Z_{D}^{V}$ & - & 1 & V-dislocation bias \\
\hline$N_{\max }$ & - & 8 & Maximum cluster size \\
\hline$K_{m}$ & - & $1.8 \times 10^{4}$ & GB mobility parameter \\
\hline$K_{m_{0}}$ & - & 25 & GB mobility parameter with pinning \\
\hline$\beta$ & - & 0.3 & Action parameter \\
\hline$\delta$ & $\mathrm{nm}$ & 1 & GB thickness \\
\hline$D_{0}^{G B}$ & $\mathrm{~m}^{2} / \mathrm{s}$ & $0.27 \times 10^{-4}$ & $\begin{array}{l}\text { Self-diffusivity of tungsten } \\
\text { along grain boundaries }\end{array}$ \\
\hline$Q^{G B}$ & $\mathrm{~J} / \mathrm{mol}$ & $4 \times 10^{5}$ & Activation energy for GB mobility \\
\hline$K_{N}$ & $\# / \mathrm{m}^{2}$ & $3.16 \times 10^{17}$ & Nucleation rate constant \\
\hline$K_{a}$ & & $1 \times 10^{8}$ & Nucleation activation energy reduction \\
\hline$\mu$ & & $161 \times 10^{9}$ & Shear modulus of tungsten \\
\hline$G_{0}$ & $\mathrm{dpa} / \mathrm{s}$ & $1 \times 10^{-6}$ & Displacement damage rate \\
\hline$r_{I V}$ & & 4.65 & Recombination radius \\
\hline$E_{T}$ & $\mathrm{~J} / \mathrm{m}^{3}$ & $5 \times 10^{5}$ & $\begin{array}{l}\text { Threshold volumetric stored energy for } \\
\text { entering the HD-set }\end{array}$ \\
\hline
\end{tabular}

Reference

Table 7: Parameter values 


\section{NIRX results}

The temporal evolution of the grain radius $r$ and stored volumetric energy $E^{V}$ during neutroninduced recrytallization at the temperatures $T=1000{ }^{\circ} \mathrm{C}, 1100{ }^{\circ} \mathrm{C}, 1200{ }^{\circ} \mathrm{C}$ and $1400{ }^{\circ} \mathrm{C}$ are shown in Figure 11 and 12.

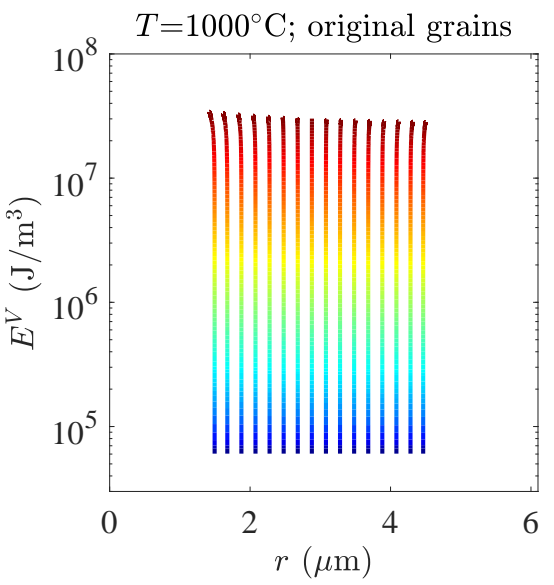

(a)

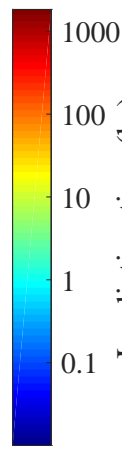

6

$10^{-1}$

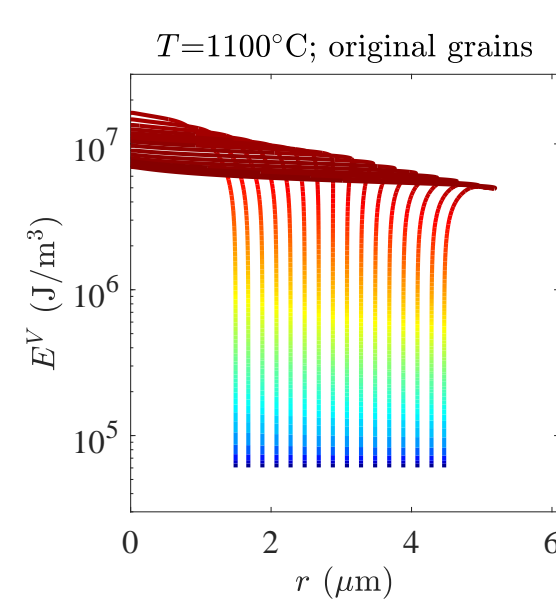

(c)

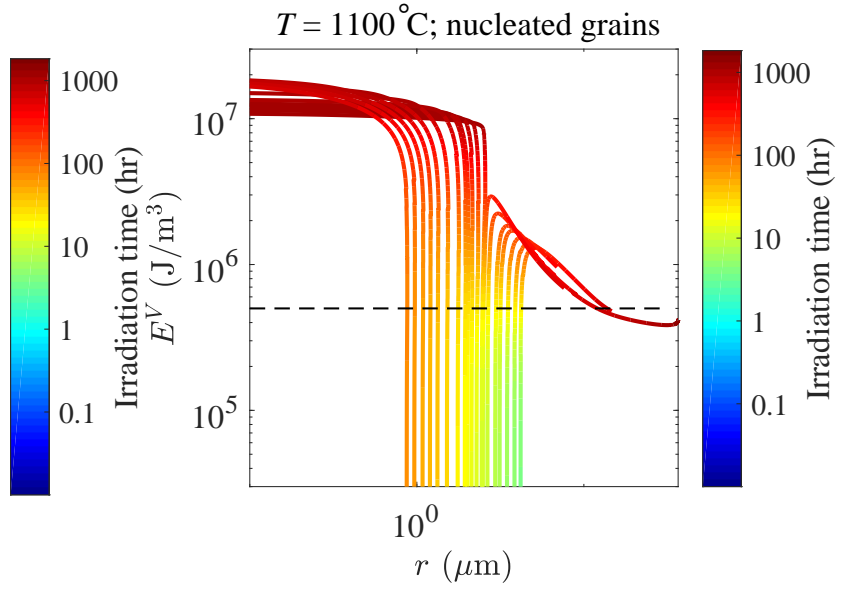

(d)

Figure 11: Temporal evolution of the grain sizes and stored defect energies for selected original grains and nucleated grains at irradiation temperatures of $1000{ }^{\circ} \mathrm{C}$ and $1100{ }^{\circ} \mathrm{C}$. 


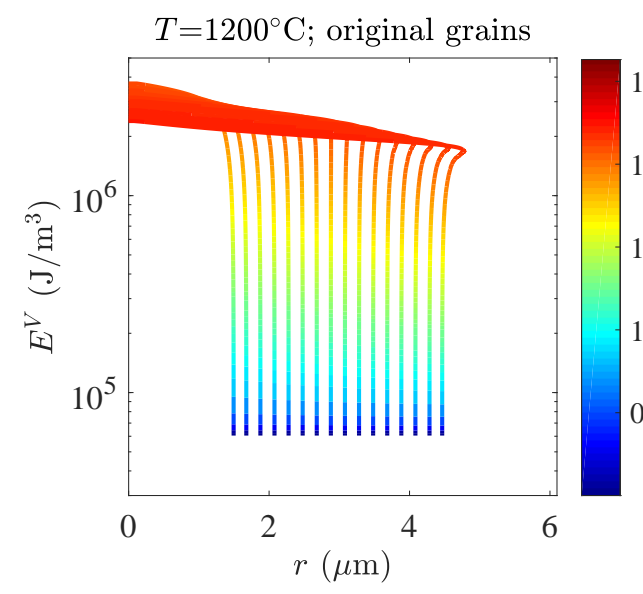

(a)

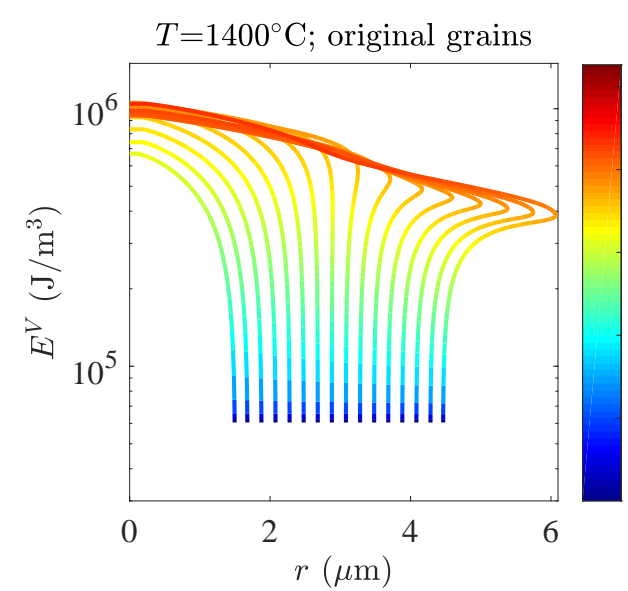

(c)

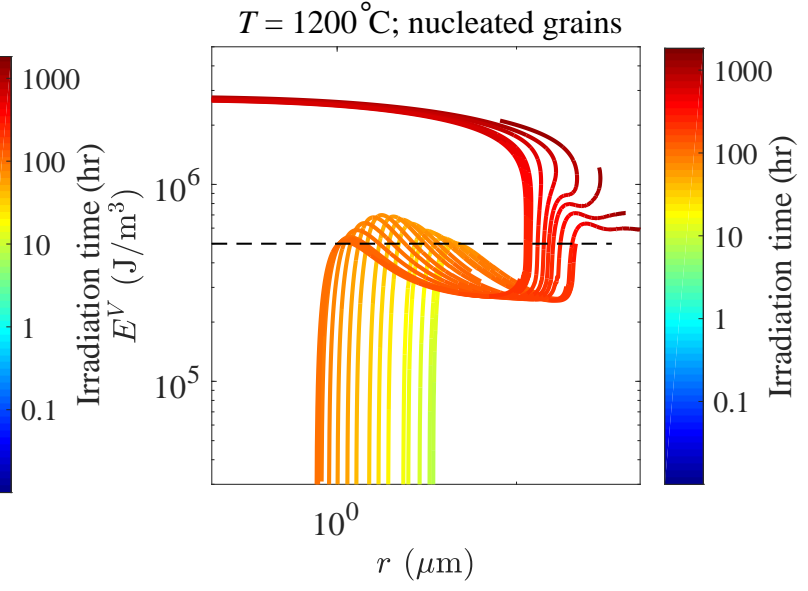

(b)

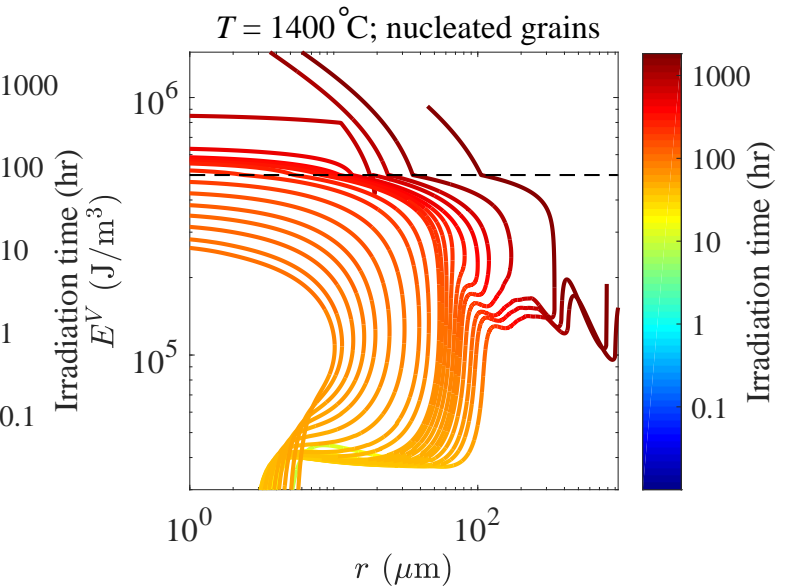

(d)

Figure 12: Temporal evolution of the grain sizes and stored defect energies for selected original grains and nucleated grains at irradiation temperatures of $1200{ }^{\circ} \mathrm{C}$ and $1400{ }^{\circ} \mathrm{C}$. 
[1] G. Janeschitz, K. Borrass, G. Federici, Y. Igitkhanov, A. Kukushkin, H. D. Pacher, G. W. Pacher, M. Sugihara. "The ITER divertor concept". Journal of Nuclear Materials (1995), 73-88. ISSN: 00223115. DOI: 10.1109/FUSION.1995.534191.

[2] R. A. Pitts, S. Carpentier, F. Escourbiac, T. Hirai, V. Komarov, S. Lisgo, A.S. Kukushkin, A. Loarte, M. Merola, A. Sashala Naik, R. Mitteau, M. Sugihara, B. Bazylev, P. C. Stangeby. "A full tungsten divertor for ITER: Physics issues and design status". Journal of Nuclear Materials (2013), S48-S56. ISSN: 00223115. DOI: $10.1016 / j$. jnucmat.2013.01.008.

[3] P. Gavila, B. Riccardi, S. Constans, J. L. Jouvelot, I. B. Vastra, M. Missirlian, M. Richou. "High heat flux testing of mock-ups for a full tungsten ITER divertor". $F u$ sion Engineering and Design (2011), 1652-1655. ISSN: 09203796. DOI: 10.1016/ j . fusengdes .2011.02.012.

[4] T. Hirai, G. Pintsuk, J. Linke, M. Batilliot. "Cracking failure study of ITER-reference tungsten grade under single pulse thermal shock loads at elevated temperatures". Journal of Nuclear Materials (2009), 751-754. ISSN: 00223115. DOI: $10.1016 / \mathrm{j}$. jnucmat.2009.01.313.

[5] S. Pestchanyi, I. Garkusha, I. Landman. "Simulation of tungsten armour cracking due to small ELMs in ITER". Fusion Engineering and Design (2010), 1697-1701. ISSN: 0920-3796. DOI: 10.1016/j.fusengdes .2010.05.005.

[6] T. Loewenhoff, J. Linke, G. Pintsuk, R. A. Pitts, B. Riccardi. "ITER-W monoblocks under high pulse number transient heat loads at high temperature". Journal of Nuclear Materials (2015), 202-205. ISSN: 0022-3115. DOI: 10.1016/j.jnucmat.2014.11.002.

[7] J. H. You. "A review on two previous divertor target concepts for DEMO mutual impact between structural design requirements and materials performance". Nuclear Fusion (2015), 113026. ISSN: 0029-5515. DOI: 10.1088/0029-5515/55/11/113026.

[8] Y. G. Li, W. H. Zhou, R. H. Ning, L. F. Huang, Z. Zeng, X. Ju. "A cluster dynamics model for accumulation of helium in tungsten under helium ions and neutron irradiation". Communications in Computational Physics (2012), 1547-1568.

[9] D. Kaoumi, A. T. Motta, R. C. Birtcher. "A thermal spike model of grain growth under irradiation". (2015). DOI: 10.1063/1.2988142.

[10] F. Haessner, H. P. Holzer. "The effect of fast neutron irradiation on the recrystallization of cold-rolled oriented copper crystals". (1954).

[11] W. V. Vaidya, K. Ehrlich. "Radiation-induced recrystallization, its cause and consequences in heavy-ion irradiated 20p cold-drawn steels of type 1.4970". Journal of Nuclear Materials (1983), 149-162. ISSN: 00223115. DOI: 10.1016/0022-3115(83) 90137-X.

[12] H. J. Stepper. "Recrystallization of alpha-particle irradiated tungsten". Metallurgical Transactions (1972), 2293-2294.

[13] O. V. Ogorodnikova, T. Płociński, M. Andrzejczuk, M. Rasiński, M. Mayer, K. J. Kurzydłowski. "TEM observations of radiation damage in tungsten irradiated by $20 \mathrm{MeV}$ W ions". Nuclear Instruments and Methods in Physics Research, Section B (2013), 159-164.

[14] E. Lassner, W. Schubert. The element tungsten. Springer, 1999.

[15] A. Lopez, D. Juul Jensen, G. N. Luo, W. Pantleon. "Recrystallization kinetics of warm-rolled tungsten in the temperature range $1150-1350^{\circ} \mathrm{C}$ ". Journal of Nuclear Materials (2014), 591-594.

[16] C. J. M. Denissen, J. Liebe, M. Van Rijswick. "Recrystallisation temperature of tungsten as a function of the heating ramp". (2006), 321-324. DOI: $10.1016 / \mathrm{j}$. ijrmhm. 2005.10.012. 
[17] F. J. Humphreys, M. Hatherly. Recrystallization and related annealing phenomena. Elsevier, 2004.

[18] M. Seita, A. Reiser, R. Spolenak. "Ion-induced grain growth and texturing in refractory thin films - A low temperature process". Applied Physics Letters (2012). ISSN: 00036951. DOI: $10.1063 / 1.4772640$.

[19] H. A. Atwater, C. V. Thompson, H. I. Smith. "Ion-bombardment-enhanced grain growth in germanium, silicon, and gold thin films". (1988).

[20] J. Rest. "Derivation of analytical expressions for the network dislocation density, change in lattice parameter, and for the recrystallized grain size in nuclear fuels". Journal of Nuclear Materials (2006), 150-159. DOI: 10.1016/j.jnucmat.2005.10.007.

[21] M. R. Tonks, X. Y. Liu, D. Andersson, D. Perez, A. Chernatynskiy, G. Pastore, C. R. Stanek, R. Williamson. "Development of a multiscale thermal conductivity model for fission gas in $\mathrm{UO}_{2}$ ". Journal of Nuclear Materials (2016), 89-98. ISSN: 00223115. DOI: 10.1016/j. jnucmat.2015.11.042.

[22] G. S. Was. Fundamentals of Radiation Materials Science. Springer, 2007, 839. ISBN: 9783540494713.

[23] J. Du, Y. Yuan, M. Wirtz, J. Linke, W. Liu, H. Greuner. "FEM study of recrystallized tungsten under ELM-like heat loads". Journal of Nuclear Materials (2015), 219-222. ISSN: 00223115. DOI: $10.1016 / j \cdot j$ jucmat. 2014.10.044.

[24] A. V. Babak, E. I. Uskov. "High-temperature embrittlement of tungsten". Strength of Materials (1984), 65-69.

[25] M. Wirtz, G. Cempura, J. Linke, G. Pintsuk, I. Uytdenhouwen. "Thermal shock response of deformed and recrystallised tungsten". Fusion Engineering and Design (2013), 1768-1772. ISSN: 09203796. DOI: 10.1016/j .fusengdes .2013.05.077.

[26] K. Farrell, A. C. Schaffhauser, J. O. Stiegler. "Recrystallization, grain growth and the ductile-brittle transition in tungsten sheet". Journal of the Less Common Metals (1967), 141-155. ISSN: 00225088. DOI: 10.1016/0022-5088(67)90177-4.

[27] Z. X. Zhang, D. S. Chen, W. T. Han, A. Kimura. "Irradiation hardening in pure tungsten before and after recrystallization". Fusion Engineering and Design (2015), 21032107. ISSN: 0920-3796. DOI: 10.1016/j.fusengdes.2015.06.192.

[28] D. Scheiber, R. Pippan, P. Puschnig, L. Romaner. "Ab initio calculations of grain boundaries in bcc metals". Modelling and Simulation in Materials Science and Engineering (2016), 35013. ISSN: 0965-0393. DOI: 10.1088/0965-0393/24/3/035013.

[29] T. Sakai, A. Belyakov, R. Kaibyshev, H. Miura, J. J. Jonas. "Dynamic and postdynamic recrystallization under hot, cold and severe plastic deformation conditions". Progress in Materials Science (2014), 130-207. ISSN: 0079-6425. DOI: $10.1016 / \mathrm{j}$. pmatsci.2013.09.002.

[30] A. Marchattiwar, A. Sarkar, J. K. Chakravartty, B. P. Kashyap. "Dynamic Recrystallization during Hot Deformation of 304 Austenitic Stainless Steel". (2013), 2168-2175. DOI: $10.1007 / \mathrm{s} 11665-013-0496-0$.

[31] H. Hallberg. "Approaches to Modeling of Recrystallization". (2011), 16-48. DOI: 10 . $3390 /$ met 1010016 .

[32] J. Orend, F. Hagemann, F. Klose, B. Maas, H. Palkowski. "A new unified approach for modeling hot rolling of steel". Material Science and Engineering: A (2015), 1-11. arXiv: 1407.4260 .

[33] K. Okuda, A. D. Rollett. "Monte Carlo simulation of elongated recrystallized grains in steels". Computational Materials Science (2005), 264-273. ISSN: 09270256. DOI: 10.1016/j. commatsci.2005.01.013.

[34] N. Moelans, B. Blanpain, P. Wollants. "Phase field simulations of grain growth in two-dimensional systems containing finely dispersed second-phase particles". Acta Materialia (2006), 1175-1184. DOI: 10.1016/j.actamat.2005.10.045. 
[35] M. Hillert. "On the theory of normal and abnormal grain growth". Acta Metallurgica (1965), 227-238.

[36] D. G. Cram, H. S. Zurob, Y. J. M. Brechet, C. R. Hutchinson. "Modelling discontinuous dynamic recrystallization using a physically based model for nucleation". Acta Materialia (2009), 5218-5228. ISSN: 1359-6454. DOI: 10.1016/j.actamat.2009.07.024.

[37] F. Montheillet, O. Lurdos, G. Damamme. "A grain scale approach for modeling steady-state discontinuous dynamic recrystallization". Acta Materialia (2009), 16021612. ISSN: 13596454. DOI: 10.1016/j . actamat. 2008.11.044.

[38] P. Bernard, S. Bag, K. Huang, R. E. Logé. "A two-site mean field model of discontinuous dynamic recrystallization". Materials Science and Engineering A (2011), 73577367.

[39] P. Duval, F. Louchet, J. Weiss, M. Montagnat. "On the role of long-range internal stresses on grain nucleation during dynamic discontinuous recrystallization". Materials Science and Engineering A (2012), 207-211. ISSN: 09215093. DOI: 10.1016/j.msea. 2012.03 .052$.

[40] G. Gottstein. "Dynamic recrystallization during high temperature deformation of magnesium". Materials Science and Engineering A (2008), 411-420. DOI: 10.1016/j. msea.2008.02.004.

[41] J. Rest. "A model for the influence of microstructure, precipitate pinning and fission gas behavior on irradiation-induced recrystallization of nuclear fuels". Journal of $\mathrm{Nu}$ clear Materials (2004), 175-184. ISSN: 00223115. DOI: 10.1016/j.jnucmat.2004.01. 009.

[42] W. Roberts, B. Ahlblom. "A nucleation criterion for dynamic recrystallization during hot working". Acta Metallurgica (1978), 801-813. ISSN: 00016160. DOI: 10.1016/0001$6160(78) 90030-5$.

[43] E. I. Poliak, J. J. Jonas. "A one-parameter approach to determining the critical conditions for the initiation of dynamic recrystallization". Acta Materialia (1996), 127136. ISSN: 13596454 . DOI: $10.1016 / 1359-6454$ (95) 00146-7.

[44] G. Gottstein, M. Frommert, M. Goerdeler, N. Schäfer. "Prediction of the critical conditions for dynamic recrystallization in the austenitic steel 800H". Materials Science and Engineering A (2004), 604-608. DOI: 10.1016/j.msea.2004.02.098.

[45] H. S. Zurob, J. Dunlop, Y. Bre. "Quantitative criterion for recrystallization nucleation in single-phase alloys: Prediction of critical strains and incubation times". Acta Materialia (2006), 3983-3990. DOI: 10.1016/j .actamat.2006.04.028.

[46] X. Yi, A. E. Sand, D. R. Mason, M. A. Kirk, S. G. Roberts, K. Nordlund, S. L. Dudarev. "Direct observation of size scaling and elastic interaction between nanoscale defects in collision cascades". Europhysics Letters (2015), 36001.

[47] D. E. J. Armstrong, X. Yi, E. A. Marquis, S. G. Roberts. "Hardening of self ion implanted tungsten and tungsten 5-wt pct. rhenium". Journal of Nuclear Materials (2013), 428-436.

[48] O. El-Atwani, A. Suslova, T. J. Novakowski, K. Hattar, M. Efe, S. S. Harilal, A. Hassanein. "In-situ TEM/heavy ion irradiation on ultrafine-and nanocrystalline-grained tungsten: Effect of 3MeV Si, $\mathrm{Cu}$ and W ions". Materials Characterization (2015), 6876.

[49] F. Ferroni, X. Yi, K. Arakawa, S. P. Fitzgerald, P. D. Edmondson, S. G. Roberts. "High temperature annealing of ion irradiated tungsten". Acta Materialia (2015), 380393.

[50] T. Jourdan, J. P. Crocombette. "Rate theory cluster dynamics simulations including spatial correlations within displacement cascades". Physical Review B (2012), 054113. ISSN: 1098-0121. DOI: 10.1103/PhysRevB . 86.054113. 
[51] W. Setyawan, G. Nandipati, K.J. Roche, H. L. Heinisch, B. D. Wirth, R. J. Kurtz. "Displacement cascades and defects annealing in tungsten, Part I: defect database from molecular dynamics simulations". Journal of Nuclear Materials (2015).

[52] R. E. Stoller. "Modeling dislocation evolution in irradiated alloys". Metallurgical Transactions A (1990), 1829-1837.

[53] T. Jourdan. "Influence of dislocation and dislocation loop biases on microstructures simulated by rate equation cluster dynamics". Journal of Nuclear Materials (2015), 286-301.

[54] Y. Watanabe, H. Iwakiri, N. Yoshida, K. Morishita, A. Kohyama. "Formation of interstitial loops in tungsten under helium ion irradiation: Rate theory modeling and experiment". Nuclear Instruments and Methods in Physics Research, Section B: Beam Interactions with Materials and Atoms (2007), 32-36. ISSN: 0168583X. DOI: 10.1016/ j.nimb.2006.11.008.

[55] Q. Xu, N. Yoshida, T. Yoshiie. "Accumulation of helium in tungsten irradiated by helium and neutrons". Journal of Nuclear Materials (2007), 806-811. ISSN: 00223115. DOI: $10.1016 / j \cdot j n u c m a t .2007 .03 .078$.

[56] J. Xu, J. Zhao. "First-principles study of hydrogen in perfect tungsten crystal". $N u$ clear Instruments and Methods in Physics Research Section B: Beam Interactions with Materials and Atoms (2009), 3170-3174. ISSN: 0168583X. DOI: 10 . 1016/j . nimb.2009.06.072.

[57] C. S. Becquart, C. Domain. "Modeling Microstructure and Irradiation Effects". Metallurgical and Materials Transactions A (2011), 852-870. ISSN: 1073-5623. DOI: 10 . $1007 / \mathrm{s} 11661-010-0460-7$.

[58] J. Marian, T. L. Hoang. "Modeling fast neutron irradiation damage accumulation in tungsten". Journal of Nuclear Materials (2012), 293-297. ISSN: 00223115. DOI: 10 . 1016/j.jnucmat.2012.06.019.

[59] T. Faney. "Numerical Simulations of Tungsten under Helium Irradiation". University of California, Berkely, 2013.

[60] S. I. Krasheninnikov, T. Faney, B. D. Wirth. "On helium cluster dynamics in tungsten plasma facing components of fusion". (2014). DOI: 10.1088/0029-5515/54/7/073019.

[61] T. Faney, B. D. Wirth. "Spatially dependent cluster dynamics modeling of microstructure evolution in low energy helium irradiated tungsten". Modelling and Simulation in Materials Science and Engineering (2014), 065010. ISSN: 0965-0393. DOI: 10.1088/ 0965-0393/22/6/065010.

[62] T. Faney, S. I. Krasheninnikov, B. D. Wirth. "Spatially dependent cluster dynamics model of He plasma surface interaction in tungsten for fusion relevant conditions". Nuclear Fusion (2015), 013014. ISSN: 0029-5515. DOI: 10.1088/0029-5515/55/1/ 013014.

[63] C. S. Becquart, C. Domain. "An object Kinetic Monte Carlo Simulation of the dynamics of helium and point defects in tungsten". Journal of Nuclear Materials (2009), 223227. ISSN: 00223115. DOI: 10.1016/j · jnucmat.2008.11.027.

[64] C. S. Becquart, C. Domain, U. Sarkar, A. De Backer, M. Hou. "Microstructural evolution of irradiated tungsten: Ab initio parameterisation of an OKMC model". Journal of Nuclear Materials (2010), 75-88. ISSN: 00223115. DOI: 10.1016/j.jnucmat. 2010. 06.003.

[65] D. R. Mason, X. Yi, M. A. Kirk, S. L. Dudarev. "Elastic trapping of dislocation loops in cascades in ion-irradiated tungsten foils". Journal of Physics: Condensed Matter (2014), 1-28. DOI: 10.1088/0953-8984/26/11/115701. arXiv: 1402.0689.

[66] G. Nandipati, W. Setyawan, H. L. Heinisch, K. Roche, R. Kurtz, B. D. Wirth. "Object Kinetic Monte Carlo simulations of radiation damage in neutron-irradiated tungsten part-I: neutron flux with a PKA spectrum corresponding to the high-flux isotope reactor". Journal of Nuclear Materials (2015), 338-344. 
[67] A. E. Sand, S. L. Dudarev, K. Nordlund. "High-energy collision cascades in tungsten: Dislocation loops structure and clustering scaling laws". Europhyics letters (2013), 46003.

[68] A. E. Sand. Molecular Dynamics Simulations of Primary Radiation Damage from Collision Cascades. 2015. ISBN: 9789515105851.

[69] P. M. Derlet, D. Nguyen-Manh, S. L. Dudarev. "Multiscale modeling of crowdion and vacancy defects in body-centered-cubic transition metals". Physical Review B (2007), 054107. ISSN: 1098-0121. DOI: 10.1103/PhysRevB .76. 054107.

[70] C. J. Ortiz, M. J. Caturla. "Simulation of defect evolution in irradiated materials: Role of intracascade clustering and correlated recombination". Physical Review B (2007), 184101. ISSN: 1098-0121. DOI: 10.1103/PhysRevB .75.184101.

[71] R.E. Stoller, S.I. Golubov, C. Domain, C.S. Becquart. "Mean field rate theory and object kinetic Monte Carlo: A comparison of kinetic models". Journal of Nuclear Materials (2008), 77-90. ISSN: 00223115. DOI: 10.1016/j.jnucmat.2008.08.047.

[72] B. Michaut, T. Jourdan, J. Malaplate, F. Sefta. "Cluster dynamics modeling and experimental investigation of the effect of injected interstitials". (2017), 166-176. DOI: 10.1016/j.jnucmat.2017.09.018.

[73] A. Hasegawa, M. Fukuda, S. Nogami, K. Yabuuchi. "Neutron irradiation effects on tungsten materials". Fusion Engineering and Design (2014), 1568-1572. ISSN: 09203796. DOI: 10.1016/j.fusengdes.2014.04.035.

[74] S. I. Golubov. Comprehensive Nuclear Materials. 1st. Elsevier Inc., 2012. DOI: 10. 1016/B978-0-08-056033-5.00029-X.

[75] D. Hull, D. J. Bacon. Introduction to Dislocations. 2001. ISBN: 978-0-7506-4681-9. DOI: http://dx.doi.org/10.1016/B978-075064681-9/50008-0.

[76] A. Lopez. "Thermal stability of warm-rolled tungsten". PhD Thesis. Technical University of Denmark, 2015.

[77] A. D. Rollett, G. Gottstein, L. Shvindlerman, D. Molodov. "Grain boundary mobility: a brief review". Zeitschrift für Metallkunde (2004), 226-229.

[78] J. Favre, D. Fabregue, D. Piot, N. Tang, Y. Koizumi, E. Maire, A. Chiba. "Modeling Grain Boundary Motion and Dynamic Recrystallization in Pure Metals". The Minerals, Metals and Materials Society (2013), 5861-5875.

[79] M. R. Gilbert, J. Marian, J. C. Sublet. "Energy spectra of primary knock-on atoms under neutron irradiation". Journal of Nuclear Materials (2015), 121-134.

[80] D. R. Olander. Fundamental aspects of nuclear reactor fuel elements. California: California University, 1972.

[81] E. Lassner, W. D. Schubert. Tungsten properties, chemistry, technology of the element, alloys, and chemical compounds. Springer Science \& Business Media, 2012. 